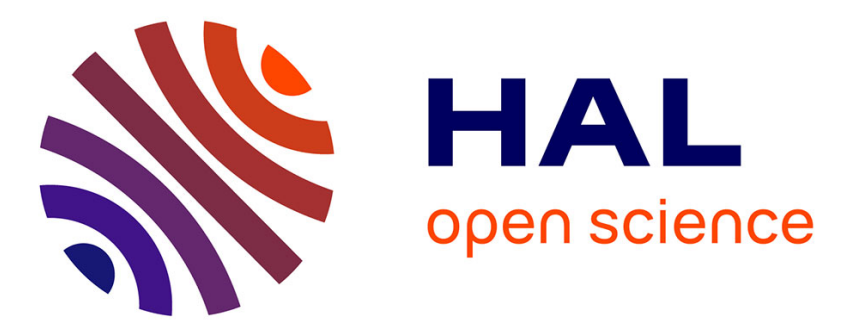

\title{
Synthesis of plant oil-based amide copolymethacrylates and their use as viscosity index improvers
}

\author{
Juliette Lomège, Violette Mohring, Vincent Lapinte, Claire Negrell, \\ Jean-Jacques Robin, Sylvain Caillol
}

\section{To cite this version:}

Juliette Lomège, Violette Mohring, Vincent Lapinte, Claire Negrell, Jean-Jacques Robin, et al.. Synthesis of plant oil-based amide copolymethacrylates and their use as viscosity index improvers. European Polymer Journal, 2018, 109, pp.435-446. 10.1016/j.eurpolymj.2018.10.015 . hal-01907227

\section{HAL Id: hal-01907227 \\ https://hal.science/hal-01907227}

Submitted on 15 May 2020

HAL is a multi-disciplinary open access archive for the deposit and dissemination of scientific research documents, whether they are published or not. The documents may come from teaching and research institutions in France or abroad, or from public or private research centers.
L'archive ouverte pluridisciplinaire HAL, est destinée au dépôt et à la diffusion de documents scientifiques de niveau recherche, publiés ou non, émanant des établissements d'enseignement et de recherche français ou étrangers, des laboratoires publics ou privés. 


\title{
Synthesis of plant oil-based amide copolymethacrylates and their use as viscosity index improvers
}

\author{
Juliette Lomège, Violette Mohring, Vincent Lapinte, Claire Negrell, Jean-Jacques \\ Robin, Sylvain Caillol* \\ Institut Charles Gerhardt Montpellier UMR 5253, Univ Montpellier CNRS ENSCM, \\ Université de Montpellier, CC1702, Place Eugène Bataillon, 34095 Montpellier Cedex 5, \\ France. \\ * Correspondence to: S. Caillol (E - mail: sylvain.caillol@enscm.fr)
}

Keywords: Fatty acids; Methacrylate; Radical polymerization; Copolymers; Lubricant; Viscosity Index Improver

\begin{abstract}
Polymeric materials derived from fatty acids (FA)s were synthesized through free radical polymerization and evaluated as viscosity index improvers (VII) in an organic triglyceride lube oil (OTO). For this purpose, various FA-based copolymers were designed to own a reduced solubility at low temperature in OTO which can be gradually improved by increasing temperature. Different fatty amide methacrylates were chosen for their poorly oil-miscible repeating units while fatty ester methacrylates, 2-(methacryloyloxy)ethyl oleate (MAEO) and 2-(methacryloyloxy)ethyl 4-(dodecylthio)oleate $\left(\mathrm{MAEO}_{\mathrm{SC} 12}\right)$, were used as oil-miscible comonomers for providing copolymers with a minimum of solubility in the lube oil. All copolymers were synthesized with a 50:50 molar feed ratio and were fully characterized through ${ }^{1} \mathrm{H}$ NMR, SEC, DSC, and TGA analyses. Then, rheological study of oil-copolymer blends revealed that copolymers containing - $\mathrm{NH}$ function were able to have a higher impact on oil viscosity at high than at low temperatures suggesting the coil copolymer expansion. This improvement of thickening power with temperature in OTO was further optimized by increasing the copolymer molecular weight, dispersity, concentration, pendant aliphatic chain length or by adding an additional aliphatic chain in the copolymer backbone. Moreover, copolymer additions in OTO did not disturb the initial Newtonian behavior of OTO at $-30{ }^{\circ} \mathrm{C}$ which corresponds to the lube oil pour point.
\end{abstract}




\section{Graphical abstract:}

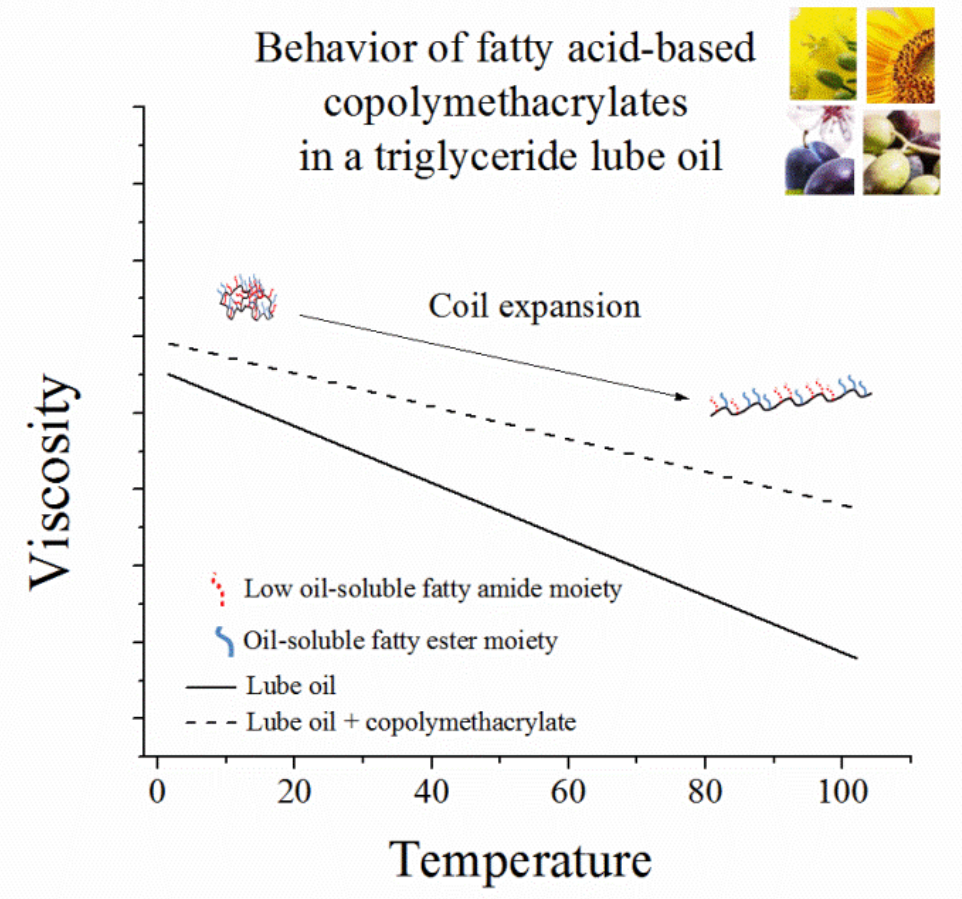

\section{Introduction}

In order to limit the decrease of viscosity with temperature, mineral oils are generally blended with viscosity index improver (VII) additives which have the ability to expand the base oil operating temperature range. ${ }^{1,2}$ These additives are well-known to reduce the lube oils viscosity-temperature dependency but also their cold flow properties. ${ }^{3}$ The main polymers used for this application are poly(alkyl)methacrylates (PMA)s. They typically have long oil soluble hydrocarbon (nonpolar) tails and smaller hydrophilic (polar) head groups which provide them specific conformation in mineral oils according to the temperature. ${ }^{4}$ Indeed, as the two parts of the polymer show different affinities in the hydrophobic base oil, it results in a coil polymer expansion with temperature inducing a limited contribution on base oil viscosity at low temperature (coil up) and a high thickening effect on base oil viscosity at high temperature (expanded coil conformation). ${ }^{5}$ Thus, the addition of PMAs in mineral oil provides, since many years, an interesting way of considerably limiting the variation of their viscosity with increasing temperature. Although the conventionally used PMAs exhibit satisfactory performances as VII additives, they originated from petroleum resources which induce environmental concerns toward their inherent toxicity. These conventional methacrylate copolymers used as viscosity modifiers in the industry are constituted of long aliphatic chain methacrylates and short aliphatic chain methacrylates which mostly come from petroleum resources (methyl methacrylate for instance). ${ }^{7,8}$ Even if some long aliphatic chain methacrylates could be obtained from fatty alcohols by oleochemistry, the industry is 
currently still using petroleum route such the Ziegler process to provide most of fatty alcohols. ${ }^{6}$ Moreover, In the meantime, the development of biolubricants are currently encouraging the design of innovative biobased VII with similar rheological properties than current PMAs in mineral oils. ${ }^{9}$ Recently, due to their specific structure with long aliphatic chains combined with a polar head, VO-based polymers resulting from direct radical polymerization have shown promising properties as VII in various mineral lube oils. ${ }^{10-12}$ However, even if they demonstrated good properties to enhance the field service performance of engine oil, VO generally showed poor reactivity in radical process. ${ }^{13}$ In most of the cases, VO required to be radically copolymerized with more reactive petrosourced comonomers to perform radical polymerization which decreases the ecological advantage of the resulting VObased additives. ${ }^{14-16}$

Hence, beyond the functionalization of VO for the synthesis of reactive building blocks for step growth polymerization, some teams have design new fatty-acid based radically polymerizable mono-functional monomers ${ }^{23}$. These new VO-based monomers allowed the preparation of biobased polyacrylates with well-defined architectures which is not possible with conventional poylcondensation. Indeed, recently, Maiti et al. reported a new method of functionalization involving Steglich esterification of various FA (caprylic, capric, lauric, myristic, palmitic, stearic and oleic acids) to provide partially biobased methacrylate monomers in a one-step method. ${ }^{19,20}$ Another synthetic route based on a two-step method through amidation followed by (meth)acrylation has been recently reported by Yuan et al. to provide monomers for radical polymerization. ${ }^{21}$ Similarly, in order to find other bioalternative, our previous work focused on the synthesis of oleic acid- based monomer, the 2-(methacryloyloxy)ethyl oleate (MAEO), which was successfully involved in free radical polymerization (FRP) and evaluated as VII. ${ }^{17}$ The resulting homopolymer (PMAEO) has demonstrated good VII properties when blended (1-10 wt. \%) in a paraffinic mineral oil (MPO). However, when PMAEO was blended in an organic biobased triglyceride oil (1-10 wt. \%), the lubricating oil acted as a too "good solvent" at low temperature which prevented the coil polymer to further expand with temperature. It resulted in a similar thickening effect on oil viscosity at low and high temperatures instead of expected VII properties. ${ }^{18}$

Hence, in order to provide suitable VII for a triglyceride oil (OTO), new copolymers were designed to promote the globular conformation in oil at low temperature and to exhibit the ability to undergo coil expansion with increasing temperature. For this purpose, lower oilsoluble comonomers were designed by using suitable functionalizations of fatty esters. Hence, this current study aims at the synthesis of new VO-based comonomers with lower solubility in OTO. In a first part, both methyl oleate and methyl erucate were functionalized by amidation with ethanolamine to add high polar amide functions which may have few affinities with the 
hydrophobic content of OTO. In addition, the methyl oleate was also functionalized with $\mathrm{N}$ methylethanolamine in order to provide a less polar derivative bearing a $\mathrm{N}$-substituted amide by a methyl group unable form H-bonds. Then, the $N$-hydroxyalkyl fatty amide derivatives were reacted with the methacrylic anhydride through trans-esterification to afford suitable monomers for free radical polymerization (FRP).

The resulting monomers were involved in copolymerization with MAEO by fixing a 50:50 feed monomers molar ratio to provide copolymers with a minimum of solubility in OTO. Additionally, further copolymers were designed under the same conditions by using another oil-soluble comonomer, the 2-(methacryloyloxy)ethyl 4-(dodecylthio)oleate (MAEO $\left.\mathrm{SC} 12_{2}\right)$ instead of MAEO. Finally, characterizations and performances of the resulting FA-based (co)polymers were evaluated as VII in OTO and then compared in terms of VII properties to the previously reported PMAEO as well as to a commercial PMA additive. The influence of several parameters such as the copolymer polarity/solubility in oil, the molecular weight, the concentration, and the aliphatic chain length were evaluated for the resulting copolymers VII properties. Additionally, cold flow behavior was also studied for the most promising FAbased copolymers as VII.

\section{Materials and methods}

\subsection{Reagents}

Methyl erucate $(80 \%)$ and methyl oleate $(83 \%)$ were kindly supplied by ITERG. Ethanolamine (99\%), N-methylethanolamine (98\%), dimethylaminopyridine (DMAP, $99 \%$ ), methacrylic anhydride (94\%), deuterated chloroform $\left(\mathrm{CDCl}_{3}\right)$ were used as received except azobis(isobutyronitrile) (AIBN, $98 \%$ ) which was recrystallized by methanol. A commercial PMA-based viscosity index improver (Viscoplex 10-250) was purchased at Evonik. All solvents including tetrahydrofuran (THF, $99 \%)$, toluene (99\%), methanol (MeOH, $99 \%)$ were purchased from VWR and were used without further purification. Organic biobased triglyceride oil (OTO) were kindly supplied by ITERG. The lubricating oil characteristics are detailed in Table 1.

Table 1: Organic triglyceride oil (OTO)

\begin{tabular}{|c|c|}
\hline \multicolumn{2}{|c|}{ Properties } \\
\hline Density at $2{ }^{\circ} \mathbf{C}\left(\right.$ g.cm $\left.^{-3}\right)$ & 0.9273 \\
\hline
\end{tabular}




\begin{tabular}{|c|c|}
\hline Kinematic viscosity at $40{ }^{\circ} \mathbf{C}\left(\mathrm{mm}^{2} \cdot \mathbf{s}^{-1}\right)$ & $26.5^{*}$ \\
\hline Kinematic viscosity at $\mathbf{1 0 0}{ }^{\circ} \mathbf{C}\left(\mathbf{m m}^{2} \cdot \mathbf{s}^{-1}\right)$ & $5.58^{*}$ \\
\hline Viscosity Index & 156 \\
\hline Pour Point $\left({ }^{\circ} \mathbf{C}\right)$ & -30 \\
\hline
\end{tabular}

*: calculated through rheological measurements (see instrumentation part below).

\subsection{Instrumentations}

Nuclear magnetic resonance (NMR) spectroscopy

${ }^{1} \mathrm{H}$ NMR spectra were acquired using a Bruker Avance $400 \mathrm{MHz}$ spectrometer equipped with a QNP z-gradient probe at room temperature. NMR samples were prepared as follows: $10 \mathrm{mg}$ of product for ${ }^{1} \mathrm{H}$ experiment in around $0.4 \mathrm{~mL}$ of $\mathrm{CDCl}_{3}$. The chemical shifts were reported in part per million ( $\mathrm{ppm}$ ) relative to tetramethylsilane. Spin multiplicity is expressed by $\mathrm{s}=$ singlet, $\mathrm{d}=$ doublet, $\mathrm{t}=$ triplet, $\mathrm{q}=$ quartet, $\mathrm{m}=$ multiplet.

\section{Size exclusion chromatography (SEC)}

Molar mass and molar mass distribution (dispersity, Đ) of polymers were determined by size exclusion chromatography using a GPC 50 Varian equipped with an RI refractive index detector. The system used two PLgel $5 \mu \mathrm{m}$ Mixed D columns with THF as eluent with a flow rate of $1 \mathrm{~mL} \cdot \mathrm{min}^{-1}$. Poly(methyl methacrylate) (PMMA) standards were used for the calibration. The typical sample concentration was $5 \mathrm{mg} \cdot \mathrm{mL}^{-1}$.

\section{Thermogravimetric analysis (TGA)}

Thermogravimetric analyses (TGA) were performed using a TGA Q50 (TA instrument) at a heating rate of $10^{\circ} \mathrm{C} \cdot \mathrm{min}^{-1}$. Approximately $10 \mathrm{mg}$ of sample were placed in an aluminum pan and heated from room temperature to $500{ }^{\circ} \mathrm{C}$ under nitrogen atmosphere $\left(60 \mathrm{~mL} \cdot \mathrm{min}^{-1}\right)$.

Differential scanning calorimetry (DSC)

Differential scanning calorimetry (DSC) analyses were carried out using a NETZSCH Maia DSC200F3 calorimeter. Constant calibration was performed using indium, $n$-octadecane and $n$-octane standards. Nitrogen was used as the purge gas. $10-15 \mathrm{mg}$ samples were sealed in aluminum pans. The thermal properties were analyzed at $20{ }^{\circ} \mathrm{C} \cdot \mathrm{min}^{-1}$ between -100 and 100 ${ }^{\circ} \mathrm{C}$.

\section{Rheometer}

Rheological investigations are executed by using a MCR 302 rheometer by Anton-Paar in plate-plate geometry with a diameter of $\mathrm{d}=25 \mathrm{~mm}$. The dynamic viscosity of OTO and OTOpolymer blends was measured from 0 to $100{ }^{\circ} \mathrm{C}$ at a shear rate of $100 \mathrm{~s}^{-1}$ and the relative viscosity (RV) in function of temperature was calculated by using the equation (1) below: 


$$
R V=\frac{\mu}{\mu_{0}} \quad \text { Eq. } 1
$$

where $\mu$ and $\mu_{0}$ correspond respectively to the dynamic viscosity of the polymer solution and the dynamic viscosity of the pure solvent.

The rheological cold flow behavior of oil-polymer blends was determined through measurements of shear stress $(\mathrm{Pa})$ and dynamic viscosity $\left(\mathrm{Pa} . \mathrm{s}^{-1}\right)$ at various shear rates ranging from 1 to $1000 \mathrm{~s}^{-1}$. The additive concentration used was $5 \mathrm{wt}$. \%. The value of the yield stress can be obtained by extrapoling the shear stress to zero shear rate on a linear curve of the relationship between shear rate and shear stress.

\section{Densimeter}

Density measurements of all formulations were performed on the DMA 4100M densimeter of Anton Paar at 40 and $100{ }^{\circ} \mathrm{C}$. Before starting the test, the oil-polymer blends were all stirred at $100{ }^{\circ} \mathrm{C}$ during 1 hour to ensure a good homogenization. Then, samples were cooled at the temperature of measurement and approximatively $1 \mathrm{~mL}$ is loaded into the densimeter to start the test. The kinematic viscosity of oil-polymer blends at 40 and $100{ }^{\circ} \mathrm{C}$ were then deducted by following the equation (2) below:

$$
v=\frac{\mu}{\rho} \quad \text { Eq. } 2
$$

where $v, \mu, \rho$ correspond respectively to the fluid kinematic viscosity $\left(\mathrm{mm}^{2} \cdot \mathrm{s}^{-1}\right)$, the fluid dynamic viscosity $\left(\mathrm{Pa}_{\mathrm{s}}{ }^{-1}\right)$, and the fluid density $\left(\mathrm{g} . \mathrm{cm}^{-3}\right)$. Finally, the viscosity indexes of the formulations were calculated according to the ASTM D2270-10 method by using the values of kinematic viscosity of polymer solutions at 40 and $100{ }^{\circ} \mathrm{C}$.

\subsection{Synthesis of $N$-hydroxyalkyl fatty amides}

- $\quad N$-(2-hydroxyethyl)oleamide (HEOA) was prepared as followed:

Methyl oleate ( $1 \mathrm{eq}, 0.1 \mathrm{~mol}, 30.0 \mathrm{~g}$ ) and ethanolamine (2 eq, $0.2 \mathrm{~mol}, 12.4 \mathrm{~g}$ ) were charged in bulk in a $250 \mathrm{~mL}$ two neck round bottom flask and stirred at $100{ }^{\circ} \mathrm{C}$. After $24 \mathrm{~h}$ of reaction, the complete conversion of amidation process was confirmed by ${ }^{1} \mathrm{H}$ NMR and the reaction was stopped. The crude product was poured in chloroform and washed twice with a $0.1 \mathrm{M}$ solution of acetic acid. Then, the chloroform was removed using a rotary evaporator to obtain pure HEOA (yield $=84 \%$ ). Its chemical structure was confirmed by ${ }^{1} \mathrm{H}$ NMR (Figure $1,(\mathrm{~A}))$.

HEOA: $\delta\left(\mathrm{ppm}, 400 \mathrm{MHz}, \mathrm{CDCl}_{3}\right): 6.01(\mathrm{NH}, 1 \mathrm{H}, \mathrm{s}), 5.40-5.28(\mathbf{H C}=\mathrm{CH}, 2 \mathrm{H}, \mathrm{m}), 3.73-3.71$ $\left(\mathrm{CH}_{2}-\mathrm{OH}, 2 \mathrm{H}, \mathrm{m}\right), 3.44-3.40\left(\mathrm{HN}-\mathrm{CH}_{2}, 2 \mathrm{H}, \mathrm{m}\right), 2.21\left(\mathbf{H}_{2} \mathrm{C}-\mathrm{C}=\mathrm{O}, 2 \mathrm{H}, \mathrm{t}\right), 1.97-2.01\left(\mathbf{H}_{2} \mathrm{C}-\right.$ 
$\left.\mathrm{CH}=\mathrm{CH}-\mathrm{CH}_{2}, 4 \mathrm{H}, \mathrm{m}\right), 1.59-1.66\left(\mathbf{H}_{2} \mathrm{C}-\mathrm{H}_{2} \mathrm{C}-\mathrm{C}=\mathrm{O}, 2 \mathrm{H}, \mathrm{m}\right), 1.24-1.26\left(-\left(\mathrm{CH}_{2}\right)_{6}-\mathrm{CH}_{2}-\mathrm{CH}=\mathrm{CH}-\right.$ $\left.\mathrm{CH}_{2^{-}}\left(\mathrm{CH}_{2}\right)_{4^{-}}, 2 \mathrm{OH}, \mathrm{m}\right), 0.87\left(\mathrm{CH}_{2}-\mathrm{CH}_{3}, 3 \mathrm{H}, \mathrm{t}\right)$.

- (Z)- $N$-(2-Hydroxyethyl)docos-13-enamide (HEDA) was prepared by following the same previous conditions with methyl erucate instead of methyl oleate (yield $=89 \%$ ). Its structure was confirmed by NMR ${ }^{1} \mathrm{H}$ (Figure S1, (A)).

HEDA: $\delta\left(\mathrm{ppm}, 400 \mathrm{MHz}, \mathrm{CDCl}_{3}\right)$ : 6.05-5.98 (NH, 1H,m), 5.33-5.36 (HC=CH, 2H, m), 3.72$3.75\left(\mathbf{H}_{2} \mathbf{C}-\mathrm{OH}, 2 \mathrm{H}, \mathrm{m}\right), 3.41-3.45\left(\mathrm{HN}-\mathrm{CH}_{2}, 2 \mathrm{H}, \mathrm{m}\right), 2.21\left(\mathbf{H}_{2} \mathrm{C}-\mathrm{C}=\mathrm{O}, 2 \mathrm{H}, \mathrm{t}\right), 1.99-2.04\left(\mathbf{H}_{2} \mathrm{C}-\right.$ $\left.\mathrm{CH}=\mathrm{CH}-\mathrm{CH}_{2}, 4 \mathrm{H}, \mathrm{m}\right), 1.59-1.66\left(\mathbf{H}_{2} \mathrm{C}-\mathrm{H}_{2} \mathrm{C}-\mathrm{C}=\mathrm{O}, 2 \mathrm{H}, \mathrm{m}\right), 1.26-1.30\left(-\left(\mathrm{CH}_{2}\right)_{6}-\mathrm{CH}_{2}-\mathrm{CH}=\mathrm{CH}-\right.$ $\left.\mathrm{CH}_{2}-\left(\mathrm{CH}_{2}\right)_{8}, 28 \mathrm{H}, \mathrm{m}\right), 0.87\left(\mathrm{CH}_{2}-\mathrm{CH}_{3}, 3 \mathrm{H}, \mathrm{t}\right)$.

- $\quad N$-(2-hydroxyethyl)-N-methyloleamide (HEMOA) was prepared by following the same procedure than HEOA with $\mathrm{N}$-methylethanolamine instead of ethanolamine (yield $=90$ $\%$ ). Its chemical structure was confirmed by ${ }^{1} \mathrm{H}$ NMR (Figure S2, (A)).

HEMOA: $\delta$ (ppm, $\left.400 \mathrm{MHz}, \mathrm{CDCl}_{3}\right)$ : 6.05-5.98 (NH, 1H,m), 5.32-5.34 (HC=CH, 2H, m), 3.74-3.77 ( $\left.\mathbf{H}_{2} \mathrm{C}-\mathrm{OH}, 2 \mathrm{H}, \mathrm{m}\right), 3.52-3.55\left(\mathrm{HN}-\mathrm{CH}_{2}, 2 \mathrm{H}, \mathrm{m}\right), 3.05\left(\mathrm{CH}_{3}-\mathrm{N}, 3 \mathrm{H}, \mathrm{s}\right), 2.32\left(\mathbf{H}_{2} \mathrm{C}-\right.$ $\mathrm{C}=\mathrm{O}, 2 \mathrm{H}, \mathrm{t}), 1.97-2.04\left(\mathbf{H}_{2} \mathrm{C}-\mathrm{CH}=\mathrm{CH}-\mathrm{CH}_{2}, 4 \mathrm{H}, \mathrm{m}\right), 1.58-1.65\left(\mathbf{H}_{2} \mathrm{C}-\mathrm{H}_{2} \mathrm{C}-\mathrm{C}=\mathrm{O}, 2 \mathrm{H}, \mathrm{m}\right), 1.24-$ $1.30\left(-\left(\mathrm{CH}_{2}\right)_{6}-\mathrm{CH}_{2}-\mathrm{CH}=\mathrm{CH}-\mathrm{CH}_{2}-\left(\mathrm{CH}_{2}\right)_{4}, 20 \mathrm{H}, \mathrm{m}\right), 0.87\left(\mathrm{CH}_{2}-\mathrm{CH}_{3}, 3 \mathrm{H}, \mathrm{t}\right)$.

\subsection{Synthesis of methacrylate monomers}

- Synthesis of fatty ester 2-(methacryloyloxy)ethyloleate (MAEO)

The synthesis procedure is exactly the same than described in our previous study. ${ }^{17}$

- Synthesis of fatty ester 2-(methacryloyloxy)ethyl 4-(dodecylthio)oleate

(MAEO $_{\text {SC12) }}$ was prepared in a two-step method as follows:

Oleic acid (1 eq, $0.106 \mathrm{~mol}, 30.0 \mathrm{~g}$ ) was charged in bulk in a $200 \mathrm{~mL}$ two neck round bottom flask. Then, AIBN (0.2 eq, $21 \mathrm{mmol}, 3.5 \mathrm{~g}$ ) and dodecanethiol (3 eq, $0.319 \mathrm{~mol}, 64.5$ g) was added into the reaction mixture and stirred at $70{ }^{\circ} \mathrm{C}$. After $24 \mathrm{~h}$ of reaction, a second addition of AIBN is added to the solution and the reaction is continued $24 \mathrm{~h}$ more. After the complete conversion of the intern unsaturation of oleic acid by thiol-ene coupling, the resulting oleic acid-based thiol derivative $\left(\mathrm{OA}_{\mathrm{SC} 12}\right)$ was purified through flash chromatography using silica gel as stationary phase and CY-EtOAc (95:5, v/v) as eluent (yield $=83 \%)$. Its structure was confirmed by ${ }^{1} \mathrm{H}$ NMR (Figure SI3, (A)).

OA $_{\text {SC12 }}: \delta$ (ppm, 400 MHz, $\left.\mathrm{CDCl}_{3}\right):$ 2.54-2.52 (-S-CH-, 1H, m), 2.47-2.43 (-CHz-S-, 2H, m), 2.33-2.30 (O=C-CH$, 2 \mathrm{H}, \mathrm{m}), 1.63-1.59\left(\mathbf{H}_{2} \mathrm{C}-\mathrm{H}_{2} \mathrm{C}-\mathrm{C}=\mathrm{O}, 4 \mathrm{H}, \mathrm{m}\right), 1.56-1.54\left(-\mathrm{CH}_{2}-\mathrm{CH}-\mathrm{CH}\right.$, 
4H, m), 1.30-1.26 (-( $\left.\mathrm{CH}_{2}\right)_{7}-\mathrm{CH}_{2}-\mathrm{CH}-\mathrm{CH}_{2}-\left(\mathrm{CH}_{2}\right)_{4}$ and $\left.\mathrm{S}-\mathrm{CH}_{2}-\left(\mathrm{CH}_{2}\right)_{10}-\mathrm{CH}_{3}, 42 \mathrm{H}, \mathrm{m}\right), 0.87$ ($\mathrm{CH}_{2}-\mathrm{CH}_{3}$ and $\left.\mathrm{S}-\mathrm{CH}_{2}-\left(\mathrm{CH}_{2}\right)_{10}-\mathrm{CH}_{3}, 6 \mathrm{H}, \mathrm{t}\right)$.

In a second step, $\mathrm{OA}_{\mathrm{SC} 12}$, DMAP and HEMA were put into a two neck round bottom flask with $200 \mathrm{~mL}$ of THF and stirred at room temperature. The reaction mixture was then put in an ice-water bath with a purge of $\mathrm{N}_{2}$ gas through it. In a separate beaker, DCC (350 mmol, 72.3 g) was dissolved in a minimum of THF and was added dropwise to the solution mixture. The ice-water bath was removed after the complete addition of DCC. After $24 \mathrm{~h}$, the white urea precipitate was filtered off and THF was removed by rotary evaporator. Then, the resulting ester was purified by column chromatography using silica gel as stationary phase and CYEtOAc $(95: 5, \mathrm{v} / \mathrm{v})$ as eluent to obtain pure monomer MAEO $_{\mathrm{SC} 12}$ (yield: $70 \%$ ). Its chemical structure was confirmed by ${ }^{1} \mathrm{H}$ NMR (Figure S3, (B))

MAEO $_{\text {SC12: }} \delta\left(\mathrm{ppm}, 400 \mathrm{MHz}, \mathrm{CDCl}_{3}\right): 6.13$ and $5.59\left(\mathrm{C}=\mathrm{CH}_{2}, 2 \mathrm{H}, \mathrm{s}\right), 4.35-4.32\left(\mathrm{OCH}_{2}-\right.$ $\left.\mathrm{CH}_{2} \mathrm{O}, 4 \mathrm{H}, \mathrm{m}\right), 2.54-2.52$ (-S-CH-, 1H, m), 2.47-2.43 (-CH $\left.\mathbf{C H}_{2}-\mathrm{S}-, 2 \mathrm{H}, \mathrm{m}\right), 2.33-2.30(\mathrm{O}=\mathrm{C}-$ $\left.\mathrm{CH}_{2}, 2 \mathrm{H}, \mathrm{m}\right), 1.95\left(\mathrm{CH}_{3}-\mathrm{C}, 1 \mathrm{H}, \mathrm{s}\right), 1.63-1.59\left(\mathbf{H}_{2} \mathrm{C}-\mathrm{H}_{2} \mathrm{C}-\mathrm{C}=\mathrm{O}, 4 \mathrm{H}, \mathrm{m}\right), 1.56-1.54\left(-\mathrm{CH}_{2}-\mathrm{CH}-\right.$ $\left.\mathrm{CH}_{2}, 4 \mathrm{H}, \mathrm{m}\right), 1.30-1.26\left(-\left(\mathrm{CH}_{2}\right)_{7}-\mathrm{CH}_{2}-\mathrm{CH}-\mathrm{CH}_{2}-\left(\mathrm{CH}_{2}\right)_{4}\right.$ and $\left.\mathrm{S}-\mathrm{CH}_{2}-\left(\mathrm{CH}_{2}\right)_{10}-\mathrm{CH}_{3}, 42 \mathrm{H}, \mathrm{m}\right)$, $0.87\left(-\mathrm{CH}_{2}-\mathrm{CH}_{3}\right.$ and $\left.\mathrm{S}-\mathrm{CH}_{2}-\left(\mathrm{CH}_{2}\right)_{10}-\mathrm{CH}_{3}, 6 \mathrm{H}, \mathrm{t}\right)$.

- 2-oleamidoethyl methacrylate (OAEM) was prepared as follows:

HEOA (1 eq, $0.08 \mathrm{~mol}, 25,0 \mathrm{~g}$ ), methacrylic anhydride (1 eq, $0.08 \mathrm{~mol}, 11.9 \mathrm{~g}$ ) and DMAP $(0,01 \mathrm{eq}, 0.8 \mathrm{mmol}, 94 \mathrm{mg})$ were mixed together in bulk into a $250 \mathrm{~mL}$ two neck round bottom flask equipped with a magnetic bar and purged with $\mathrm{N}_{2}$ gas for $20 \mathrm{~min}$. The reaction mixture was then put into an oil bath set at $60^{\circ} \mathrm{C}$ overnight. After complete conversion of methacrylate synthesis, $15 \mathrm{~mL}$ of deionized water was added into the mixture and stirred for 1 $\mathrm{h}$. The, the solution was poured in dichloromethane and washed with saturated solution of sodium bicarbonate. After the removing of solvent with rotary evaporator, an orange liquid, was obtained (yield $=92 \%$ ). Its chemical structure was confirmed by ${ }^{1} \mathrm{H}$ NMR (Figure 1 , (B)).

OAEM: $\delta\left(\mathrm{ppm}, 400 \mathrm{MHz}, \mathrm{CDCl}_{3}\right): 6.12-6.11$ and 5.60-5.59 $\left(\mathrm{C}=\mathrm{CH}_{2}, 2 \mathrm{H}, \mathrm{s}\right), 5.76-5.73$ $(\mathrm{NH}, 1 \mathrm{H}, \mathrm{s}), 5.35-5.32(\mathbf{H C}=\mathrm{CH}, 2 \mathrm{H}, \mathrm{m}), 4.26-4.24\left(\mathbf{H}_{2} \mathrm{C}-\mathrm{OH}, 2 \mathrm{H}, \mathrm{m}\right), 3.59-3.55\left(\mathrm{HN}-\mathrm{CH}{ }_{2}\right.$, $2 \mathrm{H}, \mathrm{m}), 2.20\left(\mathbf{H}_{2} \mathrm{C}-\mathrm{C}=\mathrm{O}, 2 \mathrm{H}, \mathrm{t}\right), 2.02-1.98\left(\mathbf{H}_{2} \mathrm{C}-\mathrm{CH}=\mathrm{CH}-\mathrm{CH}_{2}, 4 \mathrm{H}, \mathrm{m}\right), 1.94-1.92\left(\mathrm{CH}_{3}-\mathrm{C}, \mathrm{m}\right)$, 1.65-1.58 ( $\left.\mathbf{H}_{2} \mathrm{C}-\mathrm{H}_{2} \mathrm{C}-\mathrm{C}=\mathrm{O}, 2 \mathrm{H}, \mathrm{m}\right), 1.30-1.26\left(-\left(\mathrm{CH}_{2}\right)_{6}-\mathrm{CH}_{2}-\mathrm{CH}=\mathrm{CH}-\mathrm{CH}_{2}-\left(\mathrm{CH}_{2}\right)_{4-}, 20 \mathrm{H}, \mathrm{m}\right)$, $0.87\left(\mathrm{CH}_{2}-\mathrm{CH}_{3}, 3 \mathrm{H}, \mathrm{t}\right)$.

The same procedure was applied on HEDA and HEMOA to obtain respectively (Z)-2(docos-13-enamido)ethyl methacrylate (DAEM) (yield $=83 \%)$ and 2-(Nmethyloleamido)ethyl methacrylate (MOAEM) (yield= $75 \%$ ). The resulting monomers structures were confirmed by ${ }^{1} \mathrm{H}$ NMR (Figure S1, (B) and Figure S2, (B) respectively). 
DAEM: $\delta\left(\mathrm{ppm}, 400 \mathrm{MHz}, \mathrm{CDCl}_{3}\right): 6.12-6.11$ and 5.60-5.59 $\left(\mathrm{C}=\mathrm{CH}_{2}, 2 \mathrm{H}, \mathrm{s}\right), 5.76-5.73$ $(\mathrm{NH}, 1 \mathrm{H}, \mathrm{m})$, 5.35-5.33 $(\mathbf{H C}=\mathrm{CH}, 2 \mathrm{H}, \mathrm{m}), 4.26-4.24\left(\mathbf{H}_{2} \mathrm{C}-\mathrm{OH}, 2 \mathrm{H}, \mathrm{m}\right), 3.59-3.55\left(\mathrm{HN}-\mathrm{CH}_{2}\right.$, 2H,m), $2.20\left(\mathbf{H}_{2} \mathrm{C}-\mathrm{C}=\mathrm{O}, 2 \mathrm{H}, \mathrm{t}\right), 2.03-1.98\left(\mathbf{H}_{2} \mathrm{C}-\mathrm{CH}=\mathrm{CH}-\mathrm{CH}_{2}, 4 \mathrm{H}, \mathrm{m}\right), 1.94-1.92\left(\mathrm{CH}_{3}-\mathrm{C}, \mathrm{m}\right)$, 1.65-1.57 ( $\left.\mathbf{H}_{2} \mathrm{C}-\mathrm{H}_{2} \mathrm{C}-\mathrm{C}=\mathrm{O}, 2 \mathrm{H}, \mathrm{m}\right), 1.30-1.25\left(-\left(\mathrm{CH}_{2}\right)_{6}-\mathrm{CH}_{2}-\mathrm{CH}=\mathrm{CH}-\mathrm{CH}_{2}-\left(\mathrm{CH}_{2}\right)_{8-}, 28 \mathrm{H}, \mathrm{m}\right)$, $0.87\left(\mathrm{CH}_{2}-\mathrm{CH}_{3}, 3 \mathrm{H}, \mathrm{t}\right)$.

MOAEM: $\delta\left(\mathrm{ppm}, 400 \mathrm{MHz}, \mathrm{CDCl}_{3}\right):$ 6.10-6.08 and 5.57-5.55 $\left(\mathrm{C}=\mathrm{CH}_{2}, 2 \mathrm{H}, \mathrm{s}\right), 5.34-5.31$ $(\mathbf{H C}=\mathrm{CH}, 2 \mathrm{H}, \mathrm{m}), 4.29-4.25\left(\mathbf{H}_{2} \mathrm{C}-\mathrm{OH}, 2 \mathrm{H}, \mathrm{m}\right), 3.67-3.64\left(\mathrm{HN}-\mathrm{CH} \mathbf{H}_{2}, 2 \mathrm{H}, \mathrm{m}\right), 3.05\left(\mathrm{CH}_{3^{-}}\right.$ $\mathrm{N}, 3 \mathrm{H}, \mathrm{s}), 2.29\left(\mathbf{H}_{2} \mathrm{C}-\mathrm{C}=\mathrm{O}, 2 \mathrm{H}, \mathrm{t}\right), 2.05-1.97\left(\mathbf{H}_{2} \mathrm{C}-\mathrm{CH}=\mathrm{CH}-\mathrm{CH}_{2}, 4 \mathrm{H}, \mathrm{m}\right), 1.94-1.92\left(\mathrm{CH}_{3}-\mathrm{C}\right.$, m), 1.66-1.57 $\left(\mathbf{H}_{2} \mathrm{C}-\mathrm{H}_{2} \mathrm{C}-\mathrm{C}=\mathrm{O}, 2 \mathrm{H}, \mathrm{m}\right), 1.33-1.24\left(-\left(\mathrm{CH}_{2}\right)_{6}-\mathrm{CH}_{2}-\mathrm{CH}=\mathrm{CH}-\mathrm{CH}_{2}-\left(\mathrm{CH}_{2}\right)_{8}, 28 \mathrm{H}\right.$, $\mathrm{m}), 0.87\left(\mathrm{CH}_{2}-\mathrm{CH}_{3}, 3 \mathrm{H}, \mathrm{t}\right)$.

\subsection{Synthesis of (co)polymers through FRP}

\subsubsection{Homopolymers of $N$-hydroxyalkyl methacrylate fatty amides}

Homopolymerization of OAEM was carried out as follows : OAEM (7 mmol, $3 \mathrm{~g}$ ) and AIBN $\left(0.08 \mathrm{mmol}, 12 \mathrm{mg}\right.$ ) were dissolved in $10 \mathrm{~mL}$ of THF at $60{ }^{\circ} \mathrm{C}$. The reaction mixture was then added into a two necked flask equipped with a magnetic bar and purged with $\mathrm{N}_{2}$ gas for $20 \mathrm{~min}$. Then, after reaching a maximum of monomer conversion, the mixture was quenched by cooling with liquid nitrogen and purified by precipitation in $\mathrm{MeOH}$ at least two times to yield a viscous liquid. The obtained polymer (POAEM) was dried under vacuum for $5 \mathrm{~h}$ at $80{ }^{\circ} \mathrm{C}$ (yield $=82 \%$ ). Its chemical structure was confirmed by ${ }^{1} \mathrm{H}$ NMR spectroscopy (Figure 1, (C)).

POAEM: $\delta\left(\mathrm{ppm}, 400 \mathrm{MHz}, \mathrm{CDCl}_{3}\right):$ 5.35-5.33 (-HC=CH-, 2H, m), 4.03-3.48 (HN-CH $\mathbf{2}^{-}$ $\left.\mathrm{CH}_{2}-\mathrm{OH}, 4 \mathrm{H}, \mathrm{m}\right), 2.22-2.20\left(\mathbf{H}_{2} \mathrm{C}-\mathrm{C}=\mathrm{O}, 2 \mathrm{H}, \mathrm{m}\right), 2.03-2.01\left(\mathbf{H}_{2} \mathrm{C}-\mathrm{CH}=\mathrm{CH}-\mathrm{CH}_{2}, 4 \mathrm{H}, \mathrm{m}\right), 1.63-$ $1.59\left(\mathbf{H}_{2} \mathrm{C}-\mathrm{H}_{2} \mathrm{C}-\mathrm{C}=\mathrm{O}, 2 \mathrm{H}, \mathrm{m}\right), 1.29-1.26\left(-\left(\mathrm{CH}_{2}\right)_{6}-\mathrm{CH}_{2}-\mathrm{CH}=\mathrm{CH}-\mathrm{CH}_{2}-\left(\mathrm{CH}_{2}\right)_{4^{-}}, 20 \mathrm{H}, \mathrm{m}\right), 1.03-$ $0.87\left(\mathrm{C}-\mathrm{CH}_{3}, 3 \mathrm{H}, \mathrm{m}\right), 0.87\left(-\mathrm{CH}_{2}-\mathrm{CH}_{3}, 3 \mathrm{H}, \mathrm{t}\right)$.

DAEM and MOAEM monomers were polymerized by following the same conditions and were obtained in $80 \%$ and $60 \%$ yields respectively. The structures of the resulting polymers were confirmed by ${ }^{1} \mathrm{H}$ NMR (Figure S1, (C) and Figure S2 (C) respectively).

PDAEM: $\delta\left(\mathrm{ppm}, 400 \mathrm{MHz}, \mathrm{CDCl}_{3}\right):$ 5.39-5.29 $(-\mathbf{H C}=\mathrm{CH}-, 2 \mathrm{H}, \mathrm{m}), 4.03-3.48\left(\mathrm{HN}_{-} \mathrm{CH}_{2^{-}}\right.$ $\left.\mathrm{CH}_{2}-\mathrm{OH}, 4 \mathrm{H}, \mathrm{m}\right), 2.22-2.19\left(\mathbf{H}_{2} \mathrm{C}-\mathrm{C}=\mathrm{O}, 2 \mathrm{H}, \mathrm{m}\right), 2.03-1.98\left(\mathbf{H}_{2} \mathrm{C}-\mathrm{CH}=\mathrm{CH}-\mathrm{CH}_{2}, 4 \mathrm{H}, \mathrm{m}\right), 1.62-$ $1.58\left(\mathbf{H}_{2} \mathrm{C}-\mathrm{H}_{2} \mathrm{C}-\mathrm{C}=\mathrm{O}, 2 \mathrm{H}, \mathrm{m}\right), 1.29-1.26\left(-\left(\mathrm{CH}_{2}\right)_{6}-\mathrm{CH}_{2}-\mathrm{CH}=\mathrm{CH}-\mathrm{CH}_{2}-\left(\mathrm{CH}_{2}\right)_{8^{-}}, 28 \mathrm{H}, \mathrm{m}\right), 1.03-$ $0.87\left(\mathrm{C}-\mathrm{CH}_{3}, 3 \mathrm{H}, \mathrm{m}\right), 0.87\left(-\mathrm{CH}_{2}-\mathrm{CH}_{3}, 3 \mathrm{H}, \mathrm{t}\right)$. 
PMOAEM: $\delta\left(\mathrm{ppm}, 400 \mathrm{MHz}, \mathrm{CDCl}_{3}\right):$ 5.39-5.29 (-HC=CH-, 2H, m), 4.04-3.62 (HN-CH $\mathbf{2}^{-}$ $\left.\mathrm{CH}_{2}-\mathrm{OH}, 4 \mathrm{H}, \mathrm{m}\right), 3.10-2.93$ ppm $\left(\mathrm{N}-\mathrm{CH}_{3}, 3 \mathrm{H}, \mathrm{m}\right), 2.35-2.30\left(\mathbf{H}_{2} \mathrm{C}-\mathrm{C}=\mathrm{O}, 2 \mathrm{H}, \mathrm{m}\right), 2.03-1.98$ $\left(\mathbf{H}_{2} \mathrm{C}-\mathrm{CH}=\mathrm{CH}-\mathrm{CH}_{2}, 4 \mathrm{H}, \mathrm{m}\right), 1.62-1.58\left(\mathbf{H}_{2} \mathrm{C}-\mathrm{H}_{2} \mathrm{C}-\mathrm{C}=\mathrm{O}, 2 \mathrm{H}, \mathrm{m}\right), 1.29-1.26\left(-\left(\mathrm{CH}_{2}\right)_{6}-\mathrm{CH}_{2^{-}}\right.$ $\left.\mathrm{CH}=\mathrm{CH}-\mathrm{CH}_{2}-\left(\mathrm{CH}_{2}\right)_{4^{-}}, 2 \mathrm{H}, \mathrm{m}\right), 1.03-0.87\left(\mathrm{C}-\mathrm{CH}_{3}, 3 \mathrm{H}, \mathrm{m}\right), 0.87\left(-\mathrm{CH}_{2}-\mathrm{CH}_{3}, 3 \mathrm{H}, \mathrm{t}\right)$.

\subsubsection{Copolymers of $N$-hydroxyalkyl fatty amides methacrylate and fatty ester methacrylate monomers}

MAEO was copolymerized with the different methacrylate monomers including OAEM, DAEM and MOAEM with a molar ratio of 50:50 and purified under the same conditions than for the homopolymerization. The copolymerization between MAEO and OAEM was also performed by varying the amount of initiator ( 1 and $2 \%$ molar) and by using also fractional initiator additions to provide copolymers with different molecular weights and dispersities. All chemical structures were confirmed by ${ }^{1} \mathrm{H}$ NMR. (Figure 3, (A) PMAEO-co-POAEM and Figure S4, (A) and (B) for PMAEO-co-PDAEM and PMAEO-co-PMOAEM respectively).

PMAEO-co-POAEM (yields ranging from and 65 and $80 \%): \delta\left(\mathrm{ppm}, 400 \mathrm{MHz}, \mathrm{CDCl}_{3}\right.$ ): 5.39-5.29 (-CH=CH-, 4H, m), 4.24-4.16 $\left(\mathrm{OCH}_{2}-\mathrm{CH}_{2} \mathrm{O}, 4 \mathrm{H}, \mathrm{m}\right), 4.02-3.50\left(\mathrm{HN}-\mathrm{CH}_{2}-\mathrm{CH}_{2}-\right.$ $\mathrm{OH}, 4 \mathrm{H}, \mathrm{m}), 2.33-2.30\left(\mathrm{O}=\mathrm{C}-\mathrm{CH}_{2}\right.$ from ester function, $\left.2 \mathrm{H}, \mathrm{m}\right), 2.22-2.19\left(\mathbf{H}_{2} \mathrm{C}-\mathrm{C}=\mathrm{O}\right.$ from amide function, $2 \mathrm{H}, \mathrm{m}), 2.05-1.98\left(\mathbf{H}_{2} \mathrm{C}-\mathrm{CH}=\mathrm{CH}-\mathrm{CH}_{2}, 8 \mathrm{H}, \mathrm{m}\right), 1.63-1.59\left(\mathbf{H}_{2} \mathrm{C}-\mathrm{H}_{2} \mathrm{C}-\mathrm{C}=\mathrm{O}\right.$, $4 \mathrm{H}, \mathrm{m}), 1.31-1.27\left(-\left(\mathrm{CH}_{2}\right)_{6}-\mathrm{CH}_{2}-\mathrm{CH}=\mathrm{CH}-\mathrm{CH}_{2}-\left(\mathrm{CH}_{2}\right)_{4^{-}}, 40 \mathrm{H}, \mathrm{m}\right), 1.05-0.87\left(\mathrm{C}-\mathrm{CH}_{3}, 6 \mathrm{H}, \mathrm{m}\right)$, $0.87\left(-\mathrm{CH}_{2}-\mathrm{CH}_{3}, 6 \mathrm{H}, \mathrm{t}\right)$.

PMAEO-co-PDAEM (yield = $59 \%): \delta\left(\mathrm{ppm}, 400 \mathrm{MHz}, \mathrm{CDCl}_{3}\right): 5.39-5.29(-\mathrm{CH}=\mathrm{CH}-, 4 \mathrm{H}$, m), 4.25-4.15 ( $\left.\mathrm{OCH}_{2}-\mathrm{CH}_{2} \mathrm{O}, 4 \mathrm{H}, \mathrm{m}\right), 4.03-3.50\left(\mathrm{HN}-\mathrm{CH}_{2}-\mathrm{CH}_{2}-\mathrm{OH}, 4 \mathrm{H}, \mathrm{m}\right), 2.34-2.30(\mathrm{O}=\mathrm{C}-$ $\mathrm{CH}_{2}$ from ester function, $\left.2 \mathrm{H}, \mathrm{m}\right), 2.22-2.19\left(\mathbf{H}_{2} \mathrm{C}-\mathrm{C}=\mathrm{O}\right.$ from amide function, 2H, m), 2.03$1.98\left(\mathbf{H}_{2} \mathrm{C}-\mathrm{CH}=\mathrm{CH}-\mathrm{CH}_{2}, 8 \mathrm{H}, \mathrm{m}\right), 1.64-1.60\left(\mathbf{H}_{2} \mathrm{C}-\mathrm{H}_{2} \mathrm{C}-\mathrm{C}=\mathrm{O}, 4 \mathrm{H}, \mathrm{m}\right), 1.30-1.26\left(-\left(\mathrm{CH}_{2}\right)_{6}{ }^{-}\right.$ $\mathrm{CH}_{2}-\mathrm{CH}-\mathrm{CH}-\mathrm{CH}_{2}-\left(\mathrm{CH}_{2}\right)_{8^{-}}$, and $\left.-\left(\mathrm{CH}_{2}\right)_{6}-\mathrm{CH}_{2}-\mathrm{CH}=\mathrm{CH}_{-}-\mathrm{CH}_{2}-\left(\mathrm{CH}_{2}\right)_{4^{-}}, 48 \mathrm{H}, \mathrm{m}\right), 1.03-0.88(\mathrm{C}-$ $\left.\mathrm{CH}_{3}, 6 \mathrm{H}, \mathrm{m}\right), 0.88\left(-\mathrm{CH}_{2}-\mathrm{CH}_{3}, 6 \mathrm{H}, \mathrm{t}\right)$.

PMAEO-co-PMOAEM (yield $=53 \%): \delta\left(\mathrm{ppm}, 400 \mathrm{MHz}, \mathrm{CDCl}_{3}\right): 5.39-5.29(-\mathrm{CH}=\mathrm{CH}-$, $4 \mathrm{H}, \mathrm{m}), 4.25-4.15\left(\mathrm{OCH}_{2}-\mathrm{CH}_{2} \mathrm{O}, 4 \mathrm{H}, \mathrm{m}\right), 4.03-3.50$ ( $\left.\mathrm{HN}-\mathrm{CH}_{2}-\mathrm{CH}_{2}-\mathrm{OH}, 4 \mathrm{H}, \mathrm{m}\right), 3.04-2.96$ $\left.\left(\mathrm{N}-\mathrm{CH}_{3}, 3 \mathrm{H}, \mathrm{m}\right), 2.34-2.30\left(\mathrm{O}=\mathrm{C}-\mathrm{CH}_{2}\right), 4 \mathrm{H}, \mathrm{m}\right)$, , 2.03-1.98 $\left(\mathbf{H}_{2} \mathrm{C}-\mathrm{CH}=\mathrm{CH}-\mathrm{CH}_{2}, 8 \mathrm{H}, \mathrm{m}\right)$, 1.64-1.60 $\left(\mathbf{H}_{2} \mathrm{C}-\mathrm{H}_{2} \mathrm{C}-\mathrm{C}=\mathrm{O}, 4 \mathrm{H}, \mathrm{m}\right), \quad 1.30-1.26 \quad\left(-\left(\mathrm{CH}_{2}\right)_{6}-\mathrm{CH}_{2}-\mathrm{CH}=\mathrm{CH}-\mathrm{CH}_{2}-\left(\mathrm{CH}_{2}\right)_{4^{-}}, 40 \mathrm{H}\right.$, m), 1.03-0.88 (C-CH3 $, 6 \mathrm{H}, \mathrm{m}), 0.88\left(-\mathrm{CH}_{2}-\mathrm{CH}_{3}, 6 \mathrm{H}, \mathrm{t}\right)$.

MAEO $_{\mathrm{SC} 12}$ was copolymerized with OAEM and DAEM by following the protocol described for the homopolymerization. Both copolymer structures were confirmed by ${ }^{1} \mathrm{H}$ NMR (Figure 3, (B) for PMAEO $_{\mathrm{SC}_{12}-\mathrm{CO}-\mathrm{POAEM} \text { and Figure S5 for } \mathrm{PMAEO}_{\mathrm{SC} 12}-\mathrm{CO}-}$ PDAEM). 


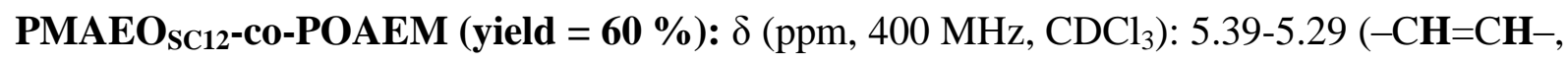
2H, m), 4.24-4.16 ( $\left.\mathrm{OCH}_{2}-\mathrm{CH}_{2} \mathrm{O}, 4 \mathrm{H}, \mathrm{m}\right), 4.02-3.50$ ( $\left.\mathrm{HN}-\mathrm{CH}_{2}-\mathrm{CH}_{2}-\mathrm{OH}, 4 \mathrm{H}, \mathrm{m}\right), 2.54-2.52$ (-

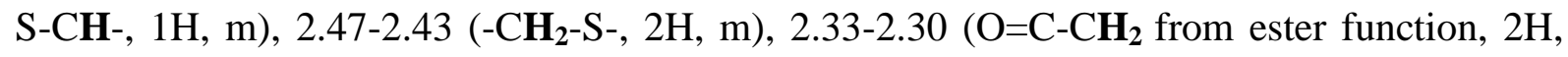
$\mathrm{m}), 2.22-2.19\left(\mathbf{H}_{2} \mathrm{C}-\mathrm{C}=\mathrm{O}\right.$ from amide function, $\left.2 \mathrm{H}, \mathrm{m}\right), 2.05-1.98\left(\mathbf{H}_{\mathbf{2}} \mathrm{C}-\mathrm{CH}=\mathrm{CH}-\mathrm{CH}_{2}, 4 \mathrm{H}\right.$, m), 1.63-1.59 ( $\left.\mathbf{H}_{2} \mathrm{C}-\mathrm{H}_{2} \mathrm{C}-\mathrm{C}=\mathrm{O}, 4 \mathrm{H}, \mathrm{m}\right), 1.56-1.54 \quad\left(-\mathrm{CH}_{2}-\mathrm{CH}-\mathrm{CH}_{2}, 4 \mathrm{H}, \mathrm{m}\right), 1.30-1.26$ ($\left(\mathrm{CH}_{2}\right)_{6}-\mathrm{CH}_{2}-\mathrm{CH}=\mathrm{CH}-\mathrm{CH}_{2}-\left(\mathrm{CH}_{2}\right)_{4}{ }^{-},-\left(\mathrm{CH}_{2}\right)_{7}-\mathrm{CH}_{2}-\mathrm{CH}_{-}-\mathrm{CH}_{2^{-}}\left(\mathrm{CH}_{2}\right)_{4}$ and and $\mathrm{S}-\mathrm{CH}_{2}-\left(\mathrm{CH}_{2}\right)_{10^{-}}$ $\left.\mathrm{CH}_{3}, 62 \mathrm{H}, \mathrm{m}\right), 1.05-0.87$ (C-CH3 $\left., 6 \mathrm{H}, \mathrm{m}\right), 0.87\left(-\mathrm{CH}_{2}-\mathrm{CH}_{3}\right.$ and $\left.\mathrm{S}-\mathrm{CH}_{2}-\left(\mathrm{CH}_{2}\right)_{10}-\mathrm{CH}_{3}, 9 \mathrm{H}, \mathrm{t}\right)$.

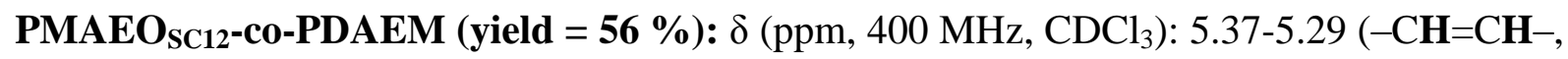
$2 \mathrm{H}, \mathrm{m}), 4.25-4.15\left(\mathrm{OCH}_{2}-\mathrm{CH}_{2} \mathrm{O}, 4 \mathrm{H}, \mathrm{m}\right), 4.00-3.50$ ( $\left.\mathrm{HN}-\mathrm{CH}_{2}-\mathrm{CH}_{2}-\mathrm{OH}, 4 \mathrm{H}, \mathrm{m}\right), 2.55-2.52$ (S-CH-, $1 \mathrm{H}, \mathrm{m}), 2.43-2.47$ (-CH $\left.\mathbf{2}_{2}-\mathrm{S}-, 2 \mathrm{H}, \mathrm{m}\right), 2.34-2.30\left(\mathrm{O}=\mathrm{C}-\mathrm{CH}_{2}\right.$ from ester function, $2 \mathrm{H}$, $\mathrm{m}), 2.22-2.19\left(\mathbf{H}_{2} \mathrm{C}-\mathrm{C}=\mathrm{O}\right.$ from amide function, $\left.2 \mathrm{H}, \mathrm{m}\right), 2.03-1.98\left(\mathbf{H}_{\mathbf{2}} \mathrm{C}-\mathrm{CH}=\mathrm{CH}-\mathrm{CH}_{2}, 4 \mathrm{H}\right.$, m), 1.64-1.60 ( $\left.\mathbf{H}_{2} \mathrm{C}-\mathrm{H}_{2} \mathrm{C}-\mathrm{C}=\mathrm{O}, 4 \mathrm{H}, \mathrm{m}\right), 1.56-1.54\left(-\mathrm{CH}_{2}-\mathrm{CH}-\mathrm{CH}_{2}, 4 \mathrm{H}, \mathrm{m}\right), 1.30-1.26$ ($\left(\mathrm{CH}_{2}\right)_{6}-\mathrm{CH}_{2}-\mathrm{CH}=\mathrm{CH}-\mathrm{CH}_{2}-\left(\mathrm{CH}_{2}\right)_{8^{-}},-\left(\mathrm{CH}_{2}\right)_{7}-\mathrm{CH}_{2}-\mathrm{CH}_{-}-\mathrm{CH}_{2^{-}}\left(\mathrm{CH}_{2}\right)_{4}$ and and $\mathrm{S}-\mathrm{CH}_{2}-\left(\mathrm{CH}_{2}\right)_{10^{-}}$ $\left.\mathrm{CH}_{3}, 70 \mathrm{H}, \mathrm{m}\right), 1.03-0.88\left(\mathrm{C}-\mathrm{CH}_{3}, 6 \mathrm{H}, \mathrm{m}\right), 0.88\left(-\mathrm{CH}_{2}-\mathrm{CH}_{3}\right.$ and $\left.\mathrm{S}-\mathrm{CH}_{2}-\left(\mathrm{CH}_{2}\right)_{10}-\mathrm{CH}_{3}, 9 \mathrm{H}, \mathrm{t}\right)$.

\section{Results and discussion}

\subsection{From fatty esters to fatty amide derivatives}

Amidation of fatty esters was carried out in bulk at $100{ }^{\circ} \mathrm{C}$ by mixing fatty esters with an excess of amino alcohol (1:2) without using catalyst. The process is generally based on consecutive transesterification and rearrangement via $\mathrm{O}-\mathrm{N}$ intramolecular acyl migration to form amides. Three fatty derivatives were prepared through this process by using different amino alcohols including ethanolamine and $N$-methylethanolamine (Scheme 1, (A)). In all the cases, the amidation of fatty esters was performed in quantitative conversion after $24 \mathrm{~h}$ of reaction. The resulting fatty amide derivatives structures were confirmed by using ${ }^{1} \mathrm{H}$ NMR spectroscopy (in $\mathrm{CDCl}_{3}$ ) where all peaks have been assigned. (Figure 1, (A) for HEOA and Figure S1, (A) and Figure S2, (A) for HEDA and HEMOA respectively). The spectra show the disappearance of the methyl protons of the ester at $3.66 \mathrm{ppm}$ and the appearance of peaks at $3.42\left(-\mathrm{NH}_{-} \mathrm{CH}_{2-}\right)$ and $3.72 \mathrm{ppm}\left(-\mathrm{CH}_{2}-\mathrm{OH}-\right)$ for HEOA and HEDA, and 3.75 and $3.54 \mathrm{ppm}$ for HEMOA corresponding to methylene protons in the $N$-hydroxyalkyl groups. The N-H peak was assigned at $6.01 \mathrm{ppm}$ while the protons of $\mathrm{N}-\mathrm{CH}_{3}$ was attributed to the peak at 3.05 ppm for HEMOA. The complete disappearance of the methyl protons at $3.66 \mathrm{ppm}$ of the fatty esters confirmed their complete conversion into $N$-hydroxyalkyl fatty amides. 
(A)

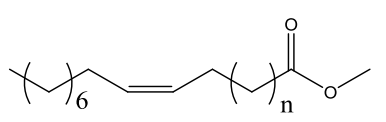

Methyl oleate $(n=6)$

Methyl erucate $(n=10)$ $\underset{100^{\circ} \mathrm{C}, \text { bulk }}{\stackrel{\text { Amino alcohol }}{\longrightarrow}}$

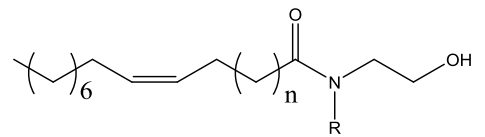

HEOA $(\mathrm{R}=-\mathrm{H}, \mathrm{n}=6)$

HEDA $(\mathrm{R}=-\mathrm{H}, \mathrm{n}=10)$

HEMOA $\left(\mathrm{R}=-\mathrm{CH}_{3}, \mathrm{n}=6\right)$

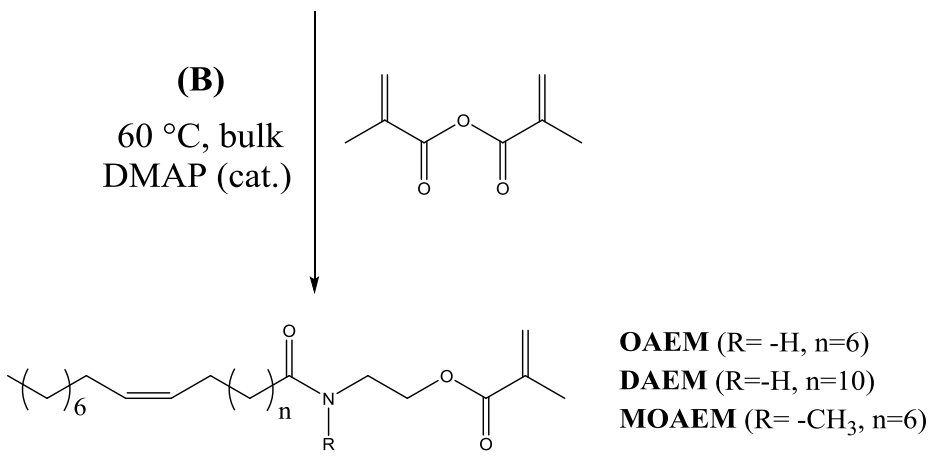

Scheme 1: Amidation of fatty esters with amino alcohols (A) and preparation of methacrylate monomers through esterification with the methacrylic anhydride with the aid of DMAP (B) 

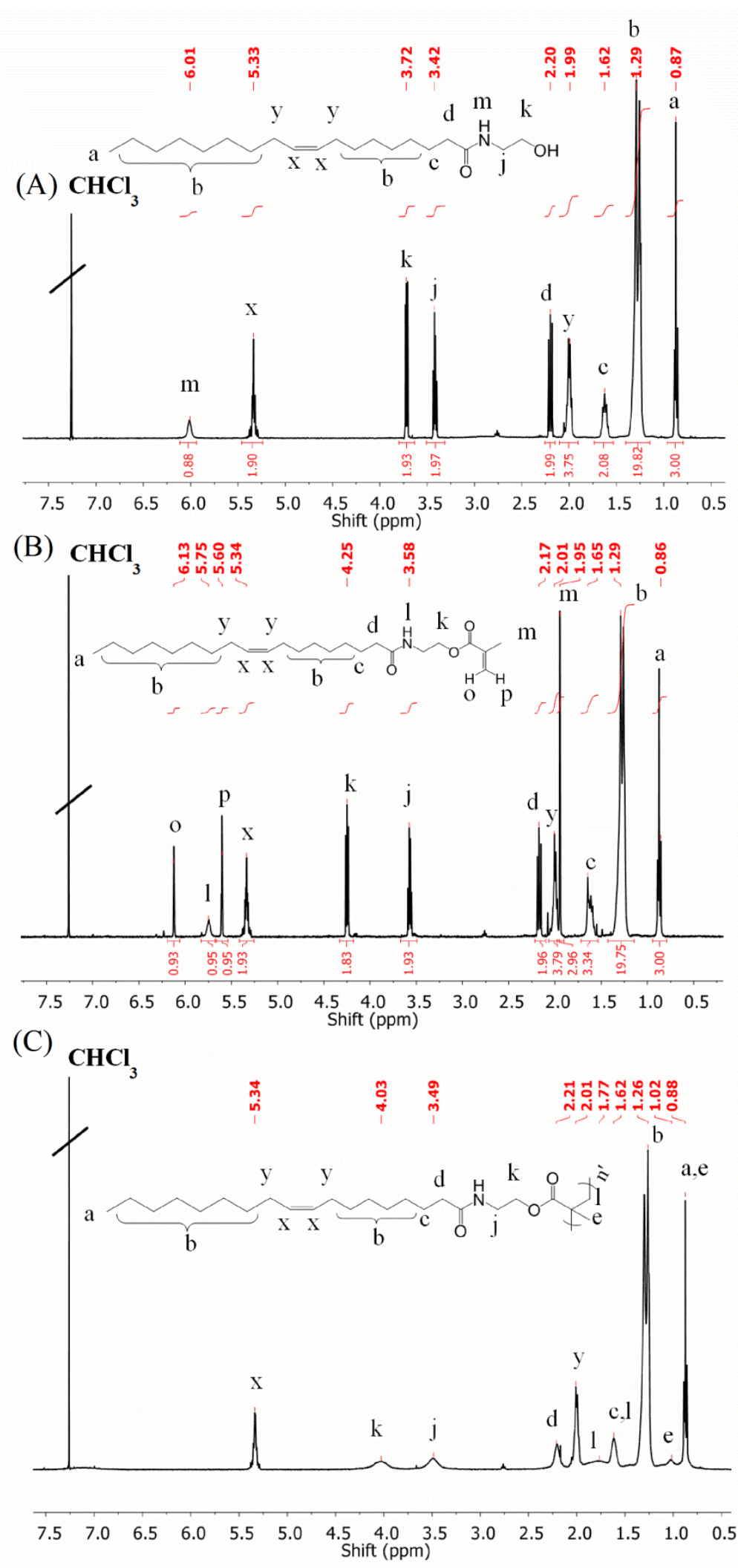

Figure 1: ${ }^{1} \mathrm{H}$ NMR spectra of HEOA (A) OAEM (B) and POAEM (C) in $\mathrm{CDCl}_{3}$

\subsection{From fatty amide derivatives to methacrylate monomers}

$N$-hydroxyalkyl fatty amides were then converted into radically polymerizable monomers using an efficient transesterification reaction catalyzed by DMAP in bulk in the presence of the methacrylic anhydride (Scheme 1, (B)). The reaction was successfully performed with 
complete conversion with a ratio $1: 1$ between the $N$-hydroxyalkyl fatty amide and the methacrylic anhydride. The methacrylation of $N$-hydroxyalkyl fatty amides was confirmed by ${ }^{1} \mathrm{H}$ NMR spectroscopy as illustrated in Figure 1, (B) for the monomer OAEM. The shift toward higher ppm of the $\alpha-\mathrm{CH}_{2}$ of amine and hydroxyl functions to respectively 3.58 and $4.25 \mathrm{ppm}$ indicated the formation of the ester group and thus, the effectiveness of the synthesis procedure. The vinylic protons of the methacrylate group were assigned to the peaks located at 6.12 and $5.60 \mathrm{ppm}$ while methyl protons next to the ester group appear at $1.95 \mathrm{ppm}$. No side reaction from the internal double bonds contained in the aliphatic chain of the methacrylate monomers was observed.

\section{3 (Co)polymerization of $N$-hydroxylalkyl fatty amide methacrylate monomers}

$N$-hydroxyalkyl fatty amide methacrylates were firstly involved in FRP in order to confirm their ability to polymerize according to a radical route. Then, they were copolymerized with the methacrylate derivative of oleic acid, the 2-(methacryloyloxy)ethyl oleate (MAEO) or the 2-(methacryloyloxy)ethyl 4-(dodecylthio)oleate $\left(\mathrm{MAEO}_{\mathrm{SC} 12}\right)$, which both are triglyceride oilsoluble monomers as previously reported. All experimental conditions and data from the resulting (co)polymers are summarized in Table 2. 
Table 2: (Co)polymerization conditions and resulting data from the (co)polymers

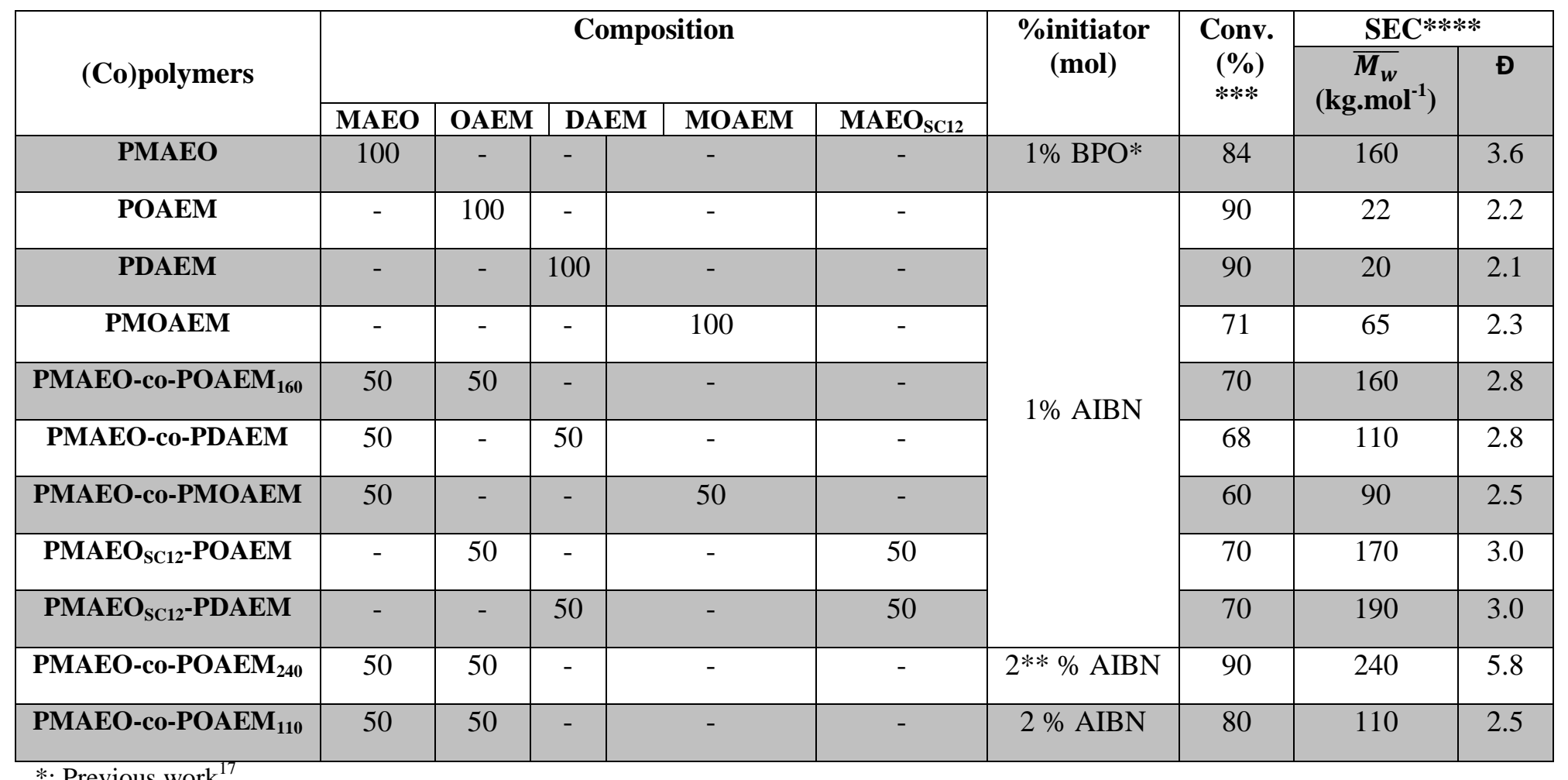

*: Previous work ${ }^{17}$

**: Successive additions of $1 \% \mathrm{~mol}$ of $\mathrm{AIBN}$ at $\mathrm{t}=0 \mathrm{~h}$ and at $\mathrm{t}=7 \mathrm{~h}$

***: Overall conversion of both monomers

****: PMMA calibration in $\mathrm{THF}$ at $35^{\circ} \mathrm{C}$ 


\subsubsection{Homopolymerization}

OAEM, DAEM and MOAEM were homopolymerized by using AIBN as radical source at $60{ }^{\circ} \mathrm{C}$ in THF as shown in Scheme 2, (A).

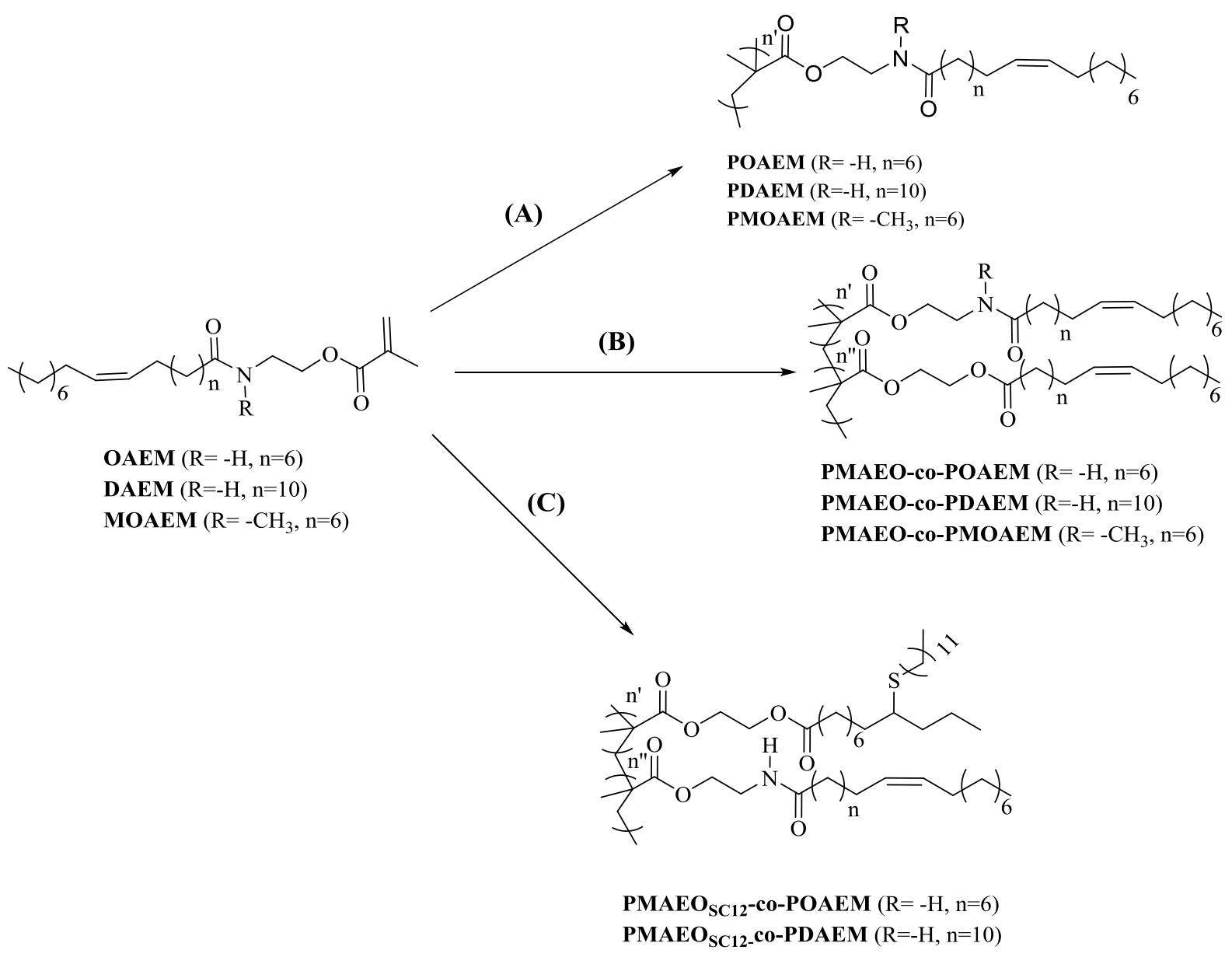

Scheme 2: Homopolymerization of $N$-hydroxyalkyl fatty methacrylate amide monomers in THF at 60 ${ }^{\circ} \mathrm{C}$ with AIBN (A), Copolymerizations of MAEO with $N$-hydroxyalkyl fatty methacrylate amide monomers in THF at $60{ }^{\circ} \mathrm{C}$ with AIBN (B), Copolymerizations of $\mathrm{MAEO}_{\mathrm{SC} 12}$ with either OAEM or DAEM in THF at $60^{\circ} \mathrm{C}$ with AIBN (C)

Polymerization of OAEM and DAEM were achieved in $90 \%$ conversion after $7 \mathrm{~h}$ of reaction which confirmed their good reactivity through radical process (Figure 2). Hence, the difference of chain length did not change the reactivity of polymerization. On the other hand, the substitution of the $\mathrm{N}$ atom by a methyl group of MOAEM reduced the reactivity by inducing more steric hindrance and thus, resulted in lower conversion (70 \%) compared to OAEM and DAEM in the same polymerization conditions. 


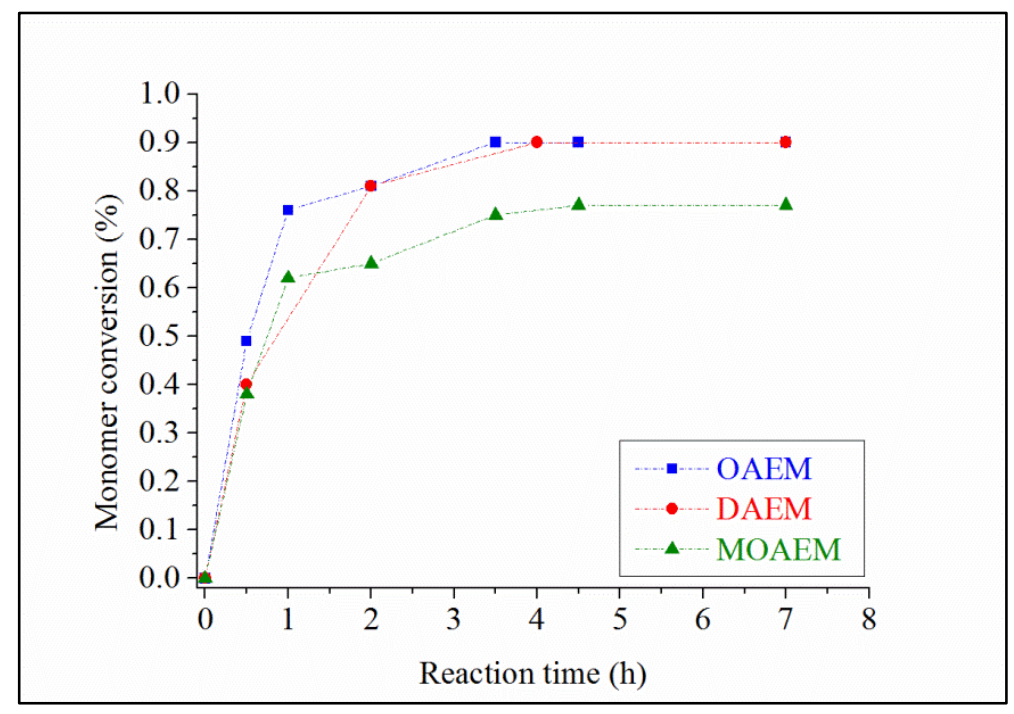

Figure 2: $N$-hydroxyalkyl fatty methacrylate amide monomer conversion (\%) vs reaction time (h)

The ${ }^{1} \mathrm{H}$ NMR spectrum of POAEM is shown in Figure 1, (C) while that of PDAEM and PMOAEM are shown in Figure S1, (C) and Figure S2, (C) respectively. In the ${ }^{1} \mathrm{H}$ NMR spectra of the resulting polymers, the disappearance of peaks assigned to the vinyl protons of the methacrylate group indicated the complete removal of unreacted monomer after precipitation in cold methanol. The methylene protons of the $N$-hydroxyalkyl groups bearing in the polymer side chain appeared at 4.04 and $3.48 \mathrm{ppm}$ while methyl protons of the polymer backbone are assigned to the peak between 1.02 and $0.88 \mathrm{ppm}$. According to the ${ }^{1} \mathrm{H}$ NMR spectra, the internal unsaturation contained in the monomer aliphatic chain remains stable during the polymerization process as no side reaction was observed. Table 2 summarizes all data related to the resulting $N$-hydroxyalkyl fatty methacrylate amides-based homopolymers including their conversions, yields and molecular weights.

\subsubsection{Copolymerization with fatty ester methacrylate monomers}

The $N$-hydroxyalkyl fatty amide methacrylates were firstly copolymerized in THF at 50:50 molar ratio with the fatty methacrylate ester, MAEO according to the homopolymerization procedure described in the experimental section (THF, $1 \%$ molar of AIBN, $60{ }^{\circ} \mathrm{C}$ ) (Scheme 2, (B)). Similarly, OAEM and DAEM were also copolymerized at 50:50 molar ratio with the highly hydrophobic oleic acid-comonomer, $\mathrm{MAEO}_{\mathrm{SC} 12}$ (Scheme 2, (C)). The resulting copolymers were obtained between 60 and $90 \%$ of conversion after $24 \mathrm{~h}$ with a lower rate than their respective homopolymers (Table 2). Figure 3 showed ${ }^{1} \mathrm{H}$ NMR spectra of PMAEO-

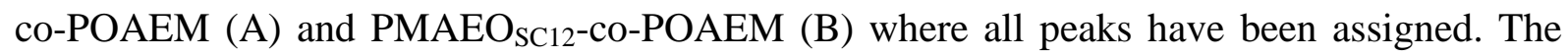


other copolymers spectra are reported in Figure S4, (A) for the copolymers PMAEO-coPDAEM, Figure S4, (B) for the copolymer PMAEO-co-PMOAEM and in Figure S5 for the

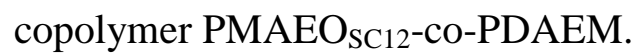

The reactivity of the comonomers through radical polymerization was monitored by ${ }^{1} \mathrm{H}$ NMR spectroscopy. In a first step, aliquots of each copolymer solution were taken at periodic time intervals and analyzed by ${ }^{1} \mathrm{H}$ NMR. Only the total monomer conversions were calculated by this method as methacrylate proton peaks of all monomers have similar shifts (6.13-6.09 and 5.60-5.56 ppm). Thus, the previous aliquots were also reprecipitated in cold methanol and analyzed through ${ }^{1} \mathrm{H}$ NMR in order to evaluate the copolymer composition at periodic times. For this purpose, the integral value of the $\alpha-\mathrm{CH}_{2}$ peak at $2.32 \mathrm{ppm}$ belonging to the ester of the poly(2-(methacryloyloxy)ethyl oleate) (PMAEO) or the poly(2-(methacryloyloxy)ethyl 4(dodecylthio)oleate) ( $\mathrm{PMAEO}_{\mathrm{SC12}}$ ) moieties was compared to that of the $\alpha-\mathrm{CH}_{2}$ peak of the amide function at $2.20 \mathrm{ppm}$ belonging to $\operatorname{poly}(N$-hydroxyalkyl fatty amide methacrylates). For copolymerizations with MOAEM, the copolymer composition was monitored by comparing the integral value of $\mathrm{CH}_{3}-\mathrm{N}$ at 3.08-2.99 ppm of PMOAEM and the ethylene protons $\left(\mathrm{CH}=\mathrm{CH}_{2}\right)$ at 5.34 ppm contained in both copolymer moieties. Hence, by knowing both the total monomer conversions and copolymer compositions at times $t$, the conversion of each monomer has been deducted. The results revealed that the FA-based monomers showed similar reactivity by radical polymerization which have induced more likely statistic copolymers. Figure S6 shows the similar kinetic reactivity between MAEO and either OAEM, DAEM or MOAEM. Moreover, despite an additional grafted alkyl chains, $\mathrm{MAEO}_{\mathrm{SC} 12}$ have demonstrated similar reactivity than OAEM and DAEM (Figure S7). Finally, all the final copolymer compositions were in accordance with the theoretical monomer feed (50:50 molar ratio).

The resulting copolymer molecular weights were measured by SEC and summarized in Table 2. Focusing on PMAEO-co-POAEM copolymers, higher molecular weights were reached by using lower initiator amounts. For instance, with 1 and $2 \%$ molar of AIBN, the weight average molecular weight attained 160 and $110 \mathrm{~kg} \cdot \mathrm{mol}^{-1}$, respectively. Additionally, the synthesis of this copolymer was also performed by adding $1 \%$ molar of AIBN at the beginning of the reaction and after $7 \mathrm{~h}$ of reaction. The resulting copolymer was characterized by an average molecular weight of $240 \mathrm{~kg} \cdot \mathrm{mol}^{-1}$ as well as a broad dispersity of 5.8. Thus, fractional initiator addition allowed to increase the heterogeneity in the chain polymer length during the polymerization. 


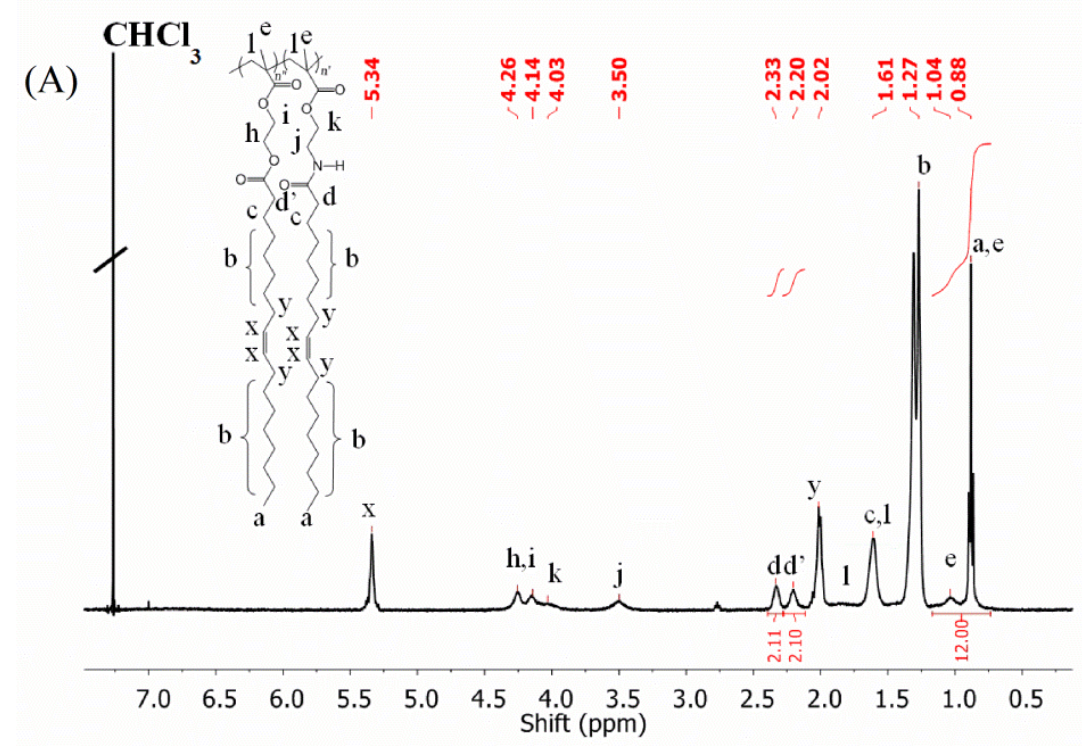

(B)

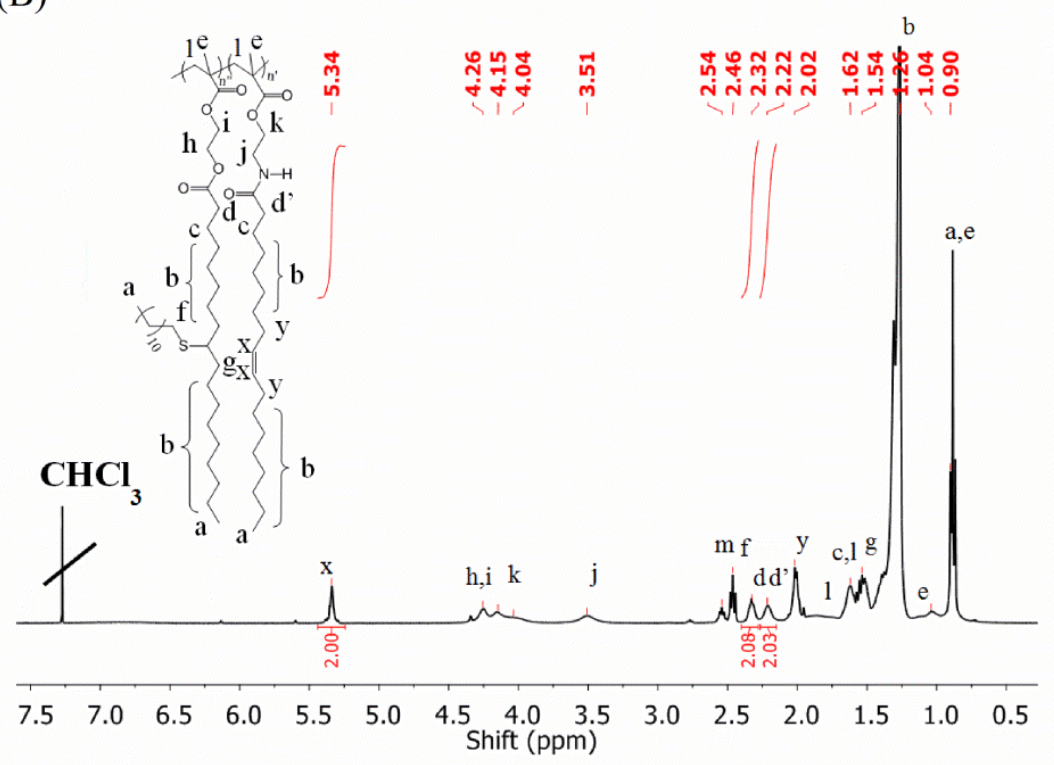

Figure 3: ${ }^{1} \mathrm{H}$ NMR spectrum of PMAEO-co-POAEM (A) and of $\mathrm{PMAEO}_{\mathrm{SC}_{12}-\mathrm{Co}-\mathrm{POAEM}(\mathrm{B}) \text { in }}$ $\mathrm{CDCl}_{3}$

\subsection{Thermal properties of the homo- and copolymers}

As the purpose of the FA-based (co)polymers is to be used as viscosity index improvers in lubricating oils, they will be frequently subjected to high local temperatures due to friction at gears, roll -contacts, bearings etc. Thus, in order to confirm their ability of being used over a wide range of temperatures, thermal properties of FA-based (co)polymers have been evaluated. The thermal degradation of homopolymers derived from $N$-hydroxyalkyl fatty 
amide methacrylates was monitored by thermogravimetric analysis (TGA) and revealed two degradation stages (Figure 4). The first degradation temperature of POAEM, PDAEM and PMOAEM occurred around $280{ }^{\circ} \mathrm{C}$ which may be related to the ester function degradation contained in the homopolymer structures. The second degradation temperature was observed around $375{ }^{\circ} \mathrm{C}$ for POAEM and PDAEM and $390{ }^{\circ} \mathrm{C}$ for PMOAEM. This latter may corresponded to the amide function degradation which is known to have a better thermal stability than ester function. ${ }^{19}$ Moreover, the higher thermal stability of PMOAEM than POAEM or PDAEM can be attributed by the substitution of nitrogen atom of amide group (N$\mathrm{CH}_{3}$ ) related to $\mathrm{N}-\mathrm{H}$ bond. In the meantime, all the FA-based copolymers showed good thermal stability up to $300{ }^{\circ} \mathrm{C}$ which is slightly higher than their analogous homopolymers (Figure S8). Hence, according to the TGA results, the methacrylate (co)polymers have shown enough thermal stability to be used as VII in lube oils operating at a temperature range below $300{ }^{\circ} \mathrm{C}$.

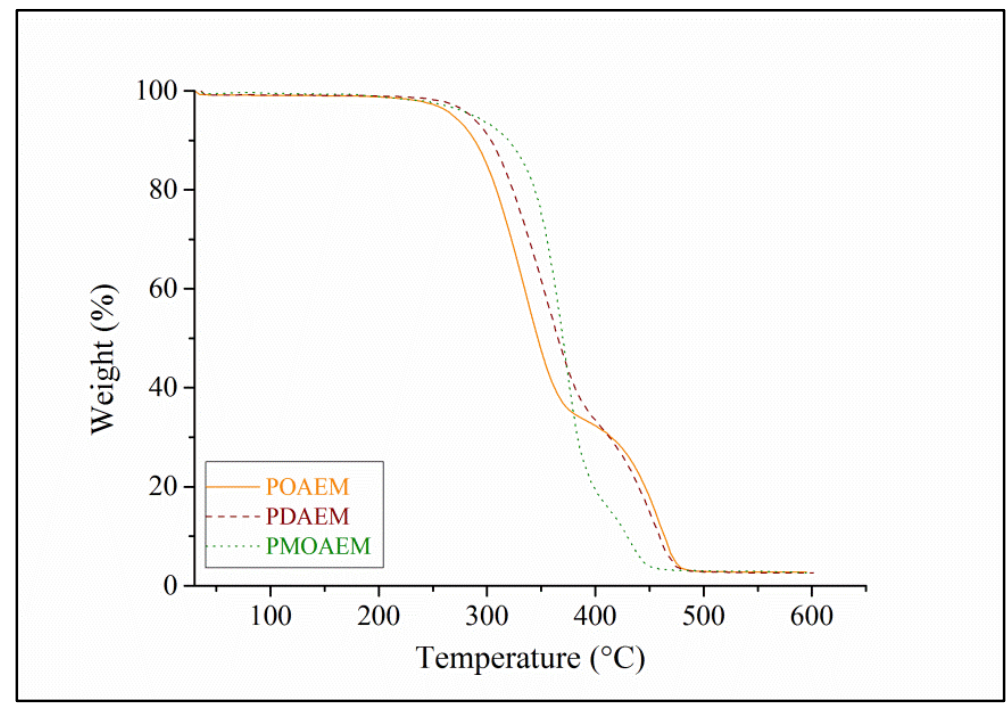

Figure 4: TGA analysis of homopolymers derived from $N$-hydroxyalkyl fatty amide methacrylate monomers

The thermal behavior of the (co)polymers and the evaluation of the glass transition temperature $\left(\mathrm{T}_{\mathrm{g}}\right)$ have been also performed by DSC analyses. The homopolymers demonstrated different $\mathrm{T}_{\mathrm{g}}$ depending on the aliphatic chain length contained in the backbone (Figure 5). For instance, a $\mathrm{T}_{\mathrm{g}}$ was observed around $-80^{\circ} \mathrm{C}$ for POAEM (C-18) and PMOAEM (C-18) while PDAEM (C-22) showed a $\mathrm{T}_{\mathrm{g}}$ at $-60{ }^{\circ} \mathrm{C}$. In a previous study, the $\mathrm{T}_{\mathrm{g}}$ of PMAEO (C-18) was noticed at $-80{ }^{\circ} \mathrm{C} .^{18}$ 


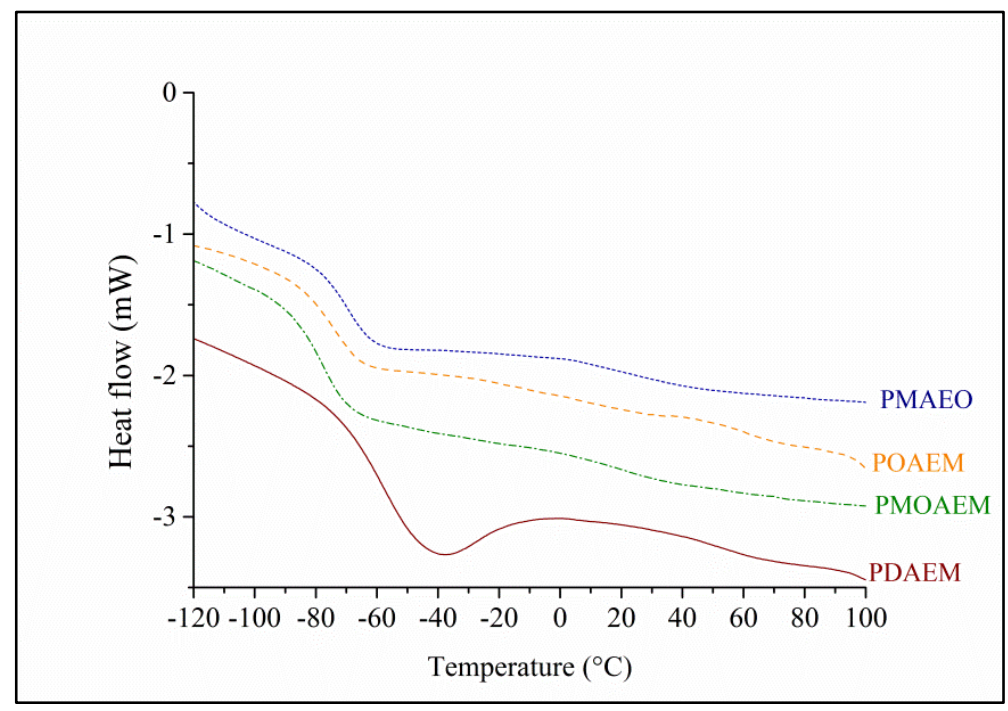

Figure 5: DSC analysis of homopolymers derived from $N$-hydroxyalkyl fatty amide methacrylatesand PMAEO

Despite their different molecular weights, copolymers PMAEO-co-POAEM have showed a similar low $\mathrm{T}_{\mathrm{g}}$ at $-80^{\circ} \mathrm{C}$. As PMAEO-co-PMOAEM has a similar chain length, its $\mathrm{T}_{\mathrm{g}}$ was also around $-80{ }^{\circ} \mathrm{C}$ while PMAEO-co-PDAEM showed a $\mathrm{T}_{\mathrm{g}}$ around $-70{ }^{\circ} \mathrm{C}$ (Figure 6). Crystalline behaviors at around $-45{ }^{\circ} \mathrm{C}$ were observed for PMAEO $\mathrm{SC} 12_{2}$-Co-POAEM and PMAEO $\mathrm{SC} 12_{2}$-COPDAEM copolymers due to their additional C-12 saturated pendant side chain. As the pour point of the lubricating oil is around $-30{ }^{\circ} \mathrm{C}$ and thus, much above the copolymer crystallization temperatures, their use as additives should not affected the oil flow behavior at cold temperature.

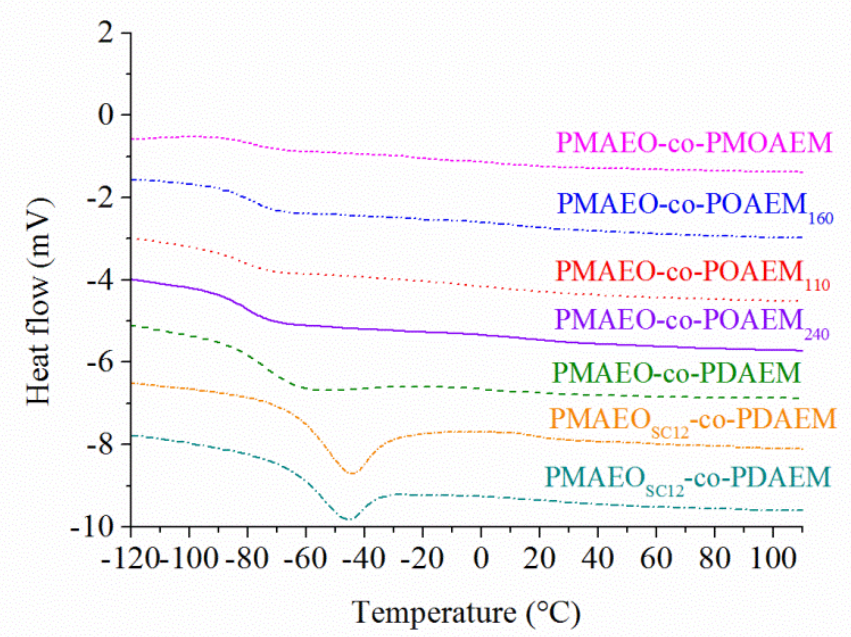

Figure 6: DSC analyses of the FA-based copolymers 


\subsection{Evaluation of the FA-based copolymers as VII}

\subsubsection{Solubility test of the resulting (co)polymers in an organic triglyceride oil (OTO)}

The resulting homopolymers derived from $N$-hydroxyalkyl fatty amide methacrylates were blended at 5 wt. \% in an organic triglyceride oil (OTO) and mixed at $100{ }^{\circ} \mathrm{C}$ for $1 \mathrm{~h}$. While PMOAEM was soluble in OTO, POAEM and PDAEM were both insoluble in the lube oil. This difference of oil affinity was related to the ability of POAEM and PDAEM to form hydrogen bonds through their -NH functions which induced high polarity and therefore made the polymers insoluble in an apolar oil such as OTO. On the other hand, substitution of the $\mathrm{NH}$ group with a -NCH3 group in PMOAEM backbone was sufficient to afford an oil-soluble polymer. Thus, even if amide is a high polar function and may decrease the polymer affinity with OTO, the long alkyl chains contained in PMOAEM are enough compatible with triglyceride compounds to obtain a minimum of solubility in OTO.

Then, the synthesized $N$-hydroxyalkyl fatty amide methacrylates have been used as low oil-soluble or oil-insoluble comonomers with either MAEO or $\mathrm{MAEO}_{\mathrm{SC} 12}$ in order to decrease the solubility of their respective homopolymers PMAEO and $\mathrm{PMAEO}_{\mathrm{SC} 12}$ in OTO. It is expected that the resulting copolymers may present a reduced solubility in OTO at low temperature and therefore it may promote the coil copolymer expansion with temperature. However, the FA-based copolymers must be a minimum soluble in oil before being evaluated as VII. Several ratios of oil-soluble and oil-insoluble monomers were evaluated for the study. When the methacrylate amide monomer content was higher than $50 \%$ molar in copolymers, the copolymers were only partially soluble or completely insoluble in the triglyceride oil even by mixing the solution at $100{ }^{\circ} \mathrm{C}$ for several hours. Therefore, as the purpose of the project was to provide copolymers with a minimum solubility in lubricating to promote its coil contraction at low temperature, the ratio 50:50 feed molar ratio (low oil-soluble moiety and oil: oil-soluble moiety) was chosen. All FA-based copolymers showed enough solubility in OTO, so they could have been evaluated as VII in the following part.

\subsubsection{Dynamic viscosity measurements of oil-polymers blends}

Rheological measurements were performed to evaluate the FA-based copolymer coil behavior in OTO with the temperature and confirm their effectiveness as VII. For this 
purpose, the relative viscosity (RV) between OTO and FA-based copolymer blends were calculated and plotted against temperature. The calculated RV represents the influence of the copolymer on oil's viscosity and should increase with the temperature in case of coil copolymer expansion. ${ }^{20}$

\subsubsection{Influence of additive concentration}

PMAEO-co-POAEM $_{240}$ copolymer was evaluated as VII in OTO with various concentrations (Figure 7). At 5 wt. \% of PMAEO-co-POAEM 240 in OTO, the RV improved by 0.55 between 0 and $100{ }^{\circ} \mathrm{C}$ while it only improved by 0.27 and 0.21 for 3 and 1 wt. $\%$ of copolymeric additive, respectively. Therefore, the greater the additive concentration, the higher the improvement of RV with temperature and the higher the copolymer thickening power. However, the use of high additive concentration in OTO also induced a non-negligible effect on the oil viscosity at low temperature. For instance, the RV was 1.92 at $0{ }^{\circ} \mathrm{C}$ with the addition of $5 \mathrm{wt} \%$ of PMAEO-co-POAEM 240 in OTO. Therefore, according to the desired application, the additive concentration must be adjusted to reach the appropriate viscosity at a specific temperature. In the following tests, $5 \mathrm{wt}$. \% of additives in OTO were used in order to evaluated the efficiency of the other copolymers as VII.

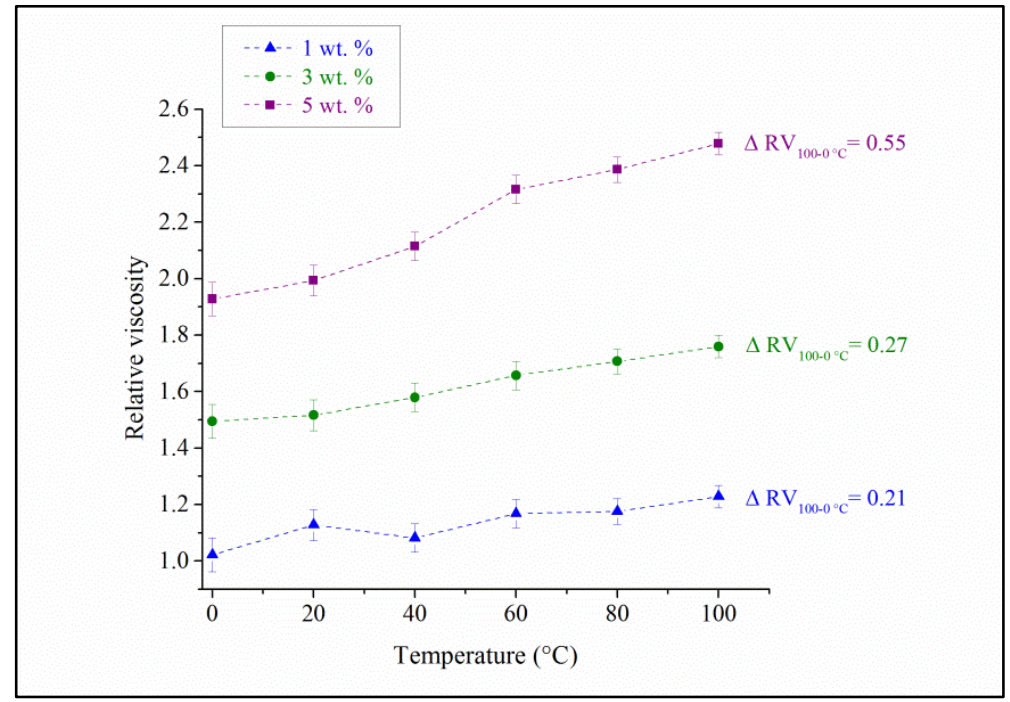

Figure 7: RV vs temperature for various PMAEO-co-POAEM ${ }_{240}$ copolymer concentrations in OTO

\subsubsection{Influence of the copolymer molecular weight and dispersity}

As illustrated in Figure 8, PMAEO-co-POAEM ${ }_{160}$ demonstrated a higher increase of RV with the temperature $\left(\Delta \mathrm{RV}_{100-0}{ }^{\circ} \mathrm{C}=0.21\right)$ compared to PMAEO-co-POAEM ${ }_{110}\left(\Delta \mathrm{RV}_{100-0}{ }^{\circ} \mathrm{C}=\right.$ 0.13). Thus, the higher the copolymer molecular weight, the higher the improvement of its 
thickening power in oil with temperature. Indeed, as mentioned in our previous work, the ability of a polymeric additive to have VII properties depends greatly of its molecular weight. ${ }^{17}$ Low polymer molecular weight generally induce a limited influence on oil viscosity either at low or high temperature which is mostly due to their reduced coil size dimension. Hence, it limits considerably the increase of the polymer thickening power with temperature and therefore its efficiency as VII. Moreover, the dispersity seems to play an important role in the properties of the copolymer as VII. As shown in Figure 8, PMAEO-co-POAEM 240 , which had a high dispersity $(\mathrm{D}=3.6)$, have demonstrated a much significant ability to reduce the viscosity-temperature dependency than both copolymers PMAEO-co-POAEM ${ }_{160}(\bigoplus=2.3)$ and PMAEO-co-POAEM $_{110}(Đ=2.3)$. However, when the molecular weight and dispersity of the additive are too high, the polymer thickening power is not negligible at low temperature in the lube oil and may disturb the oil flow. The resulting higher lube oil viscosity may therefore require more energy to put the oil in motion. However, as previously shown, the thickening power of a polymeric additive can be easily adjusted by varying its concentration in oil according to the targeted application.

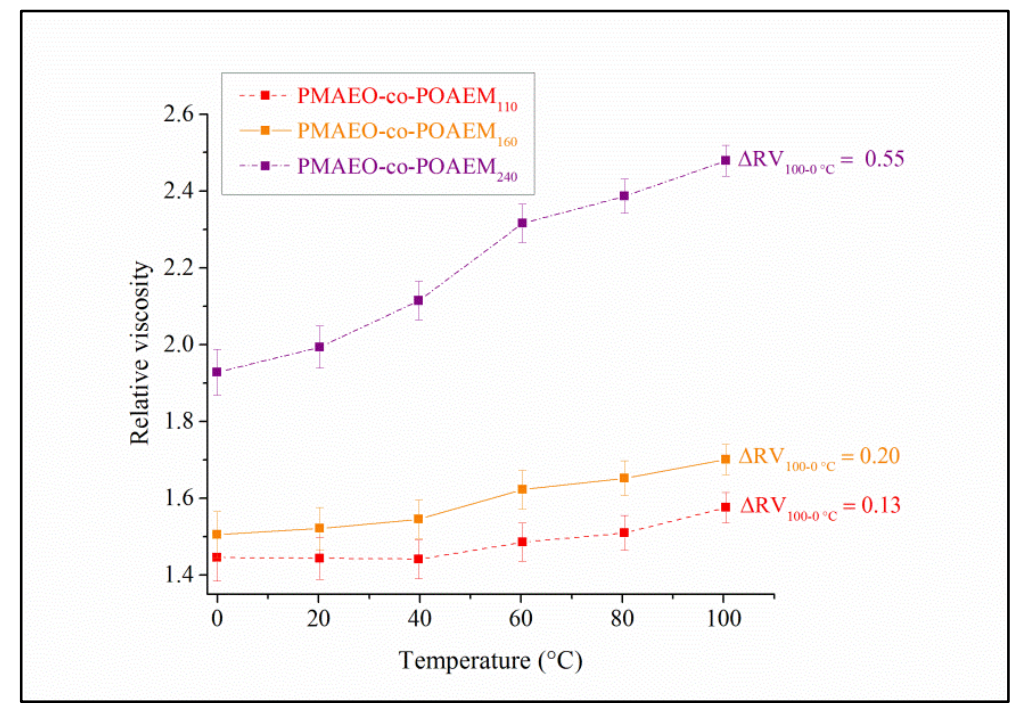

Figure 8: RV versus temperature for different molecular weights of PMAEO-co-POAEM (5 wt. \% in OTO)

\subsubsection{Influence of the copolymer composition}

The rheological FA-based copolymer properties were compared to those previously reported for PMAEO in OTO at 5 wt. \% and they were also compared to a commercial VIIbased PMA additive in this study. ${ }^{17}$ In a previous work, it was shown that PMAEO has a similar thickening power on oil viscosity at each temperature as the calculated RV between 
OTO and OTO-PMAEO blend remained constant with the increasing temperature. This observation was related to a good polymer swelling in the triglyceride oil at low temperature preventing it to further expand in oil with temperature. In this study, similar observation was noticed by using a petroleum poly(alkyl methacrylate) additive (PMA) in OTO. The RV stayed around a value of 1.50 between 0 and $100{ }^{\circ} \mathrm{C}$ meaning that even a commercial polymeric additive does not seem to be a suitable VII for OTO (Figure 9). On the opposite, the addition of PMAEO-co-POAEM ${ }_{160}$ copolymer in OTO induced an improvement of RV with the temperature. For instance, the RV between OTO and OTO-PMAEO-co-POAEM 160 blend increased from 1.50 at $0{ }^{\circ} \mathrm{C}$ to 1.70 at $100{ }^{\circ} \mathrm{C}$ meaning that the copolymer had a higher effect on oil viscosity at high temperature than at low temperature. These results underlined the better copolymer PMAEO-co-OAEM 160 ability to reduce the viscosity-temperature dependency of OTO compared to the homopolymer PMAEO. Indeed, PMAEO-co-POAEM 160 had a considerably lower contribution on OTO viscosity at $0{ }^{\circ} \mathrm{C}\left(\mathrm{RV}_{0}{ }^{\circ} \mathrm{C}=1.50\right)$ than PMAEO $\left(\mathrm{RV}_{0}{ }^{\circ} \mathrm{C}=1.70\right)$ but had a similar thickening power in $\mathrm{OTO}$ at $100{ }^{\circ} \mathrm{C}$ than PMAEO $\left(\mathrm{RV}_{100}{ }^{\circ} \mathrm{C}=\right.$ 1.70). This specific behavior may be explained by the coil copolymer expansion in oil with temperature which made the copolymer a suitable VII. On the other hand, the addition of PMAEO-co-PMOAEM in OTO didn't induce any improvement of RV with temperature since the $\mathrm{RV}$ remains around 1.55 between 0 and $100{ }^{\circ} \mathrm{C}$. The difference of $\mathrm{RV}$ tendency with temperature between the addition of PMAEO-co-POAEM ${ }_{160}$ and PMAEO-co-PMOAEM copolymers in OTO have been related to the polarity of their respective $N$-hydroxyalkyl fatty amide methacrylate moieties. As mentioned in the solubility analyzes part, POAEM was insoluble in OTO due to the formation of polar hydrogen bonds via the $-\mathrm{NH}$ group. On the contrary, as PMOAEM bearing $-\mathrm{NCH}_{3}$ group was previously found soluble in OTO, it may not be an enough low-oil soluble moiety to promote the coil expansion of PMAEOPMOAEM in OTO with temperature. 


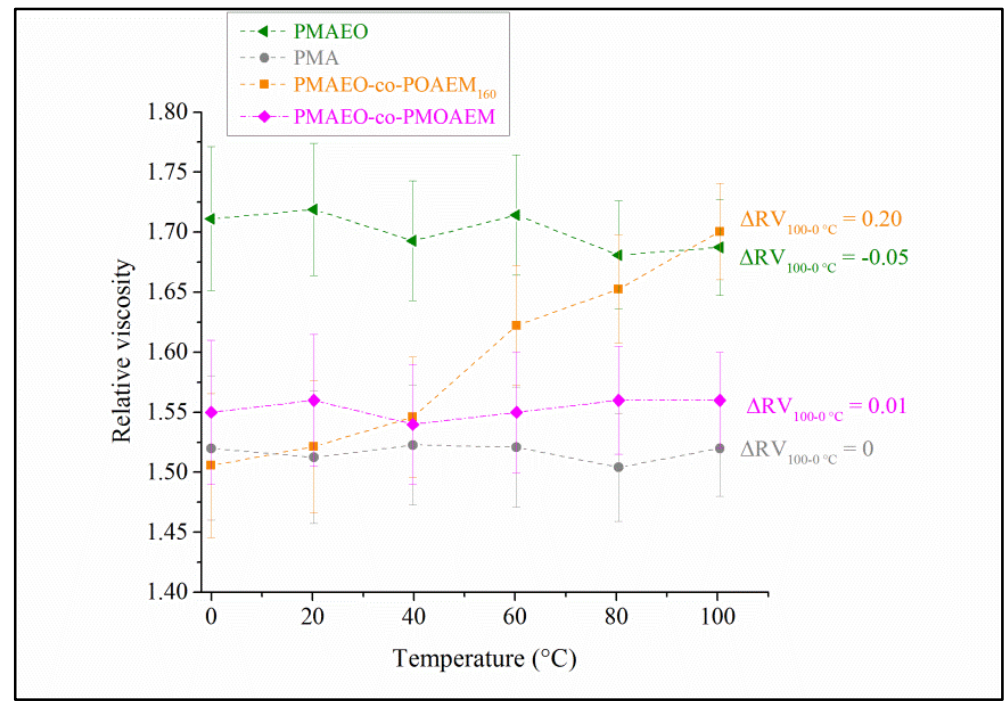

Figure 9: RV versus temperature for PMAEO, PMA, PMAEO-co-POAEM ${ }_{160}$ and PMAEO-coPMOAEM (5 wt. \% in OTO)

\subsubsection{Influence of the pendant aliphatic chain length}

The influence of the aliphatic chain length on VII additives for OTO was studied on FAbased copolymers having similar molecular weight $\left(110 \mathrm{~kg} \cdot \mathrm{mol}^{-1}\right)$ as shown in Figure 10 . The RV was increased by 0.25 between 0 and $100{ }^{\circ} \mathrm{C}$ with PMAEO-co-PDAEM (C-22) while it was only increased by 0.13 with PMAEO-co-POAEM ${ }_{110}(\mathrm{C}-18)$. This result confirmed that the increasing of the aliphatic chain length of a comonomer contained in the copolymer can promote the thickening copolymer power as well as its coil expansion with temperature. Indeed, the longer the copolymer aliphatic chain, the more copolymer-OTO interactions there are. This resulted in an improvement of the copolymer contribution on oil viscosity and more especially at high temperature when the copolymer is more likely in an expanded conformation. 


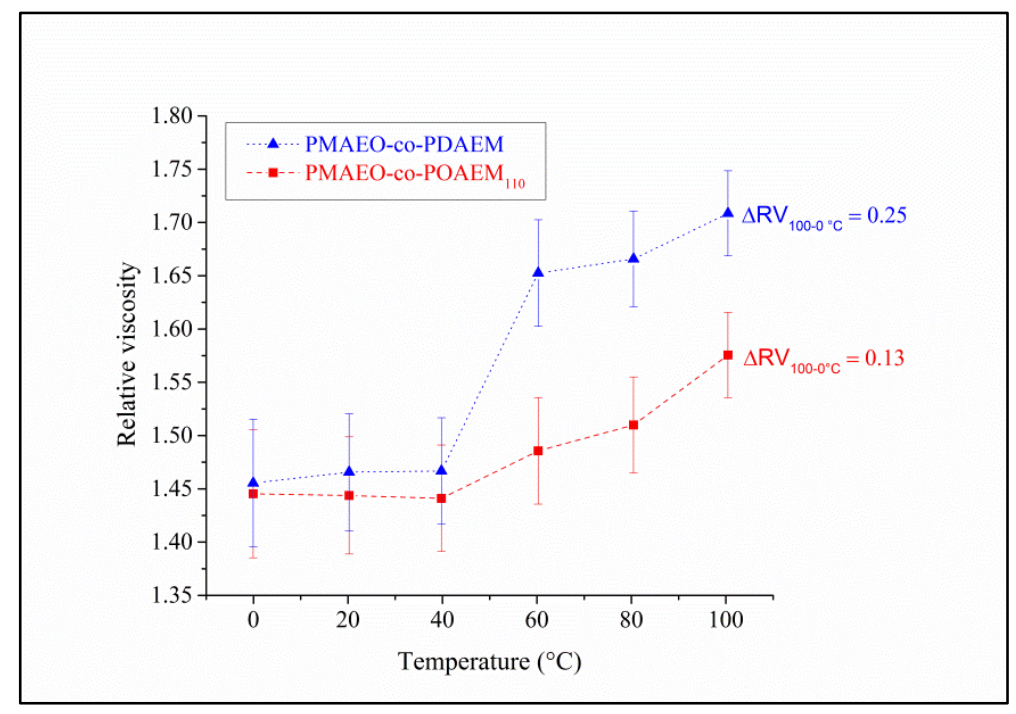

Figure 10: RV versus temperature for copolymers containing different aliphatic chain lengths (5 wt.

$\%$ in OTO)

\subsubsection{Influence of an aditionnal pendant chain length}

Copolymers containing $\mathrm{MAEO}_{\mathrm{SC} 12}$ instead of MAEO as oil-soluble comonomer and either OAEM or DAEM as oil-insoluble moieties had shown a better ability to reduce the viscositytemperature dependency of OTO between 0 and $100{ }^{\circ} \mathrm{C}$ using $5 \mathrm{wt} . \%$ of additive (Figure 11 ).

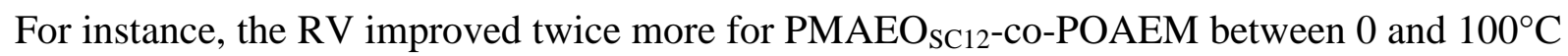
$\left(\Delta \mathrm{RV}_{100-00^{\circ} \mathrm{C}}=0.41\right)$ than for PMAEO-co-POAEM ${ }_{160}\left(\Delta \mathrm{RV}_{100-0^{\circ} \mathrm{C}}=0.20\right)$ at similar copolymer molecular weight. Moreover, oil-polymers blend containing PMAEO $_{\mathrm{SC}_{2} \text {-co- }}$ PDAEM have shown a higher improvement of $\mathrm{RV}\left(\Delta \mathrm{RV} 100-0^{\circ} \mathrm{C}=0.56\right)$ with temperature than those containing $\mathrm{PMAEO}_{\mathrm{SC} 12}$-co-POAEM $\left(\Delta \mathrm{RV}_{100-0^{\circ} \mathrm{C}}=0.41\right)$. This results confirmed the first observation about the positive aliphatic chain length influence on the coil copolymer expansion. Thus, the use of MAEOSC $_{12}$ as oil-soluble comonomer allowed to prepare copolymers with a higher thickening power improvement in OTO with temperature. In the meantime, the use of a comonomer with an additional grafted aliphatic pendant chain induced a non-negligible thickening power of copolymers in OTO at low temperature compared those containing MAEO. For example, at $0{ }^{\circ} \mathrm{C}$, the RV was 1.67 with $\mathrm{PMAEO}_{\mathrm{SC} 12}$-co-POAEM while the RV was 1.50 with PMAEO-co-POAEM 160 . As previously said, the thickening power of the polymeric additive can be easily modulated by varying its concentration in the lube oil. 


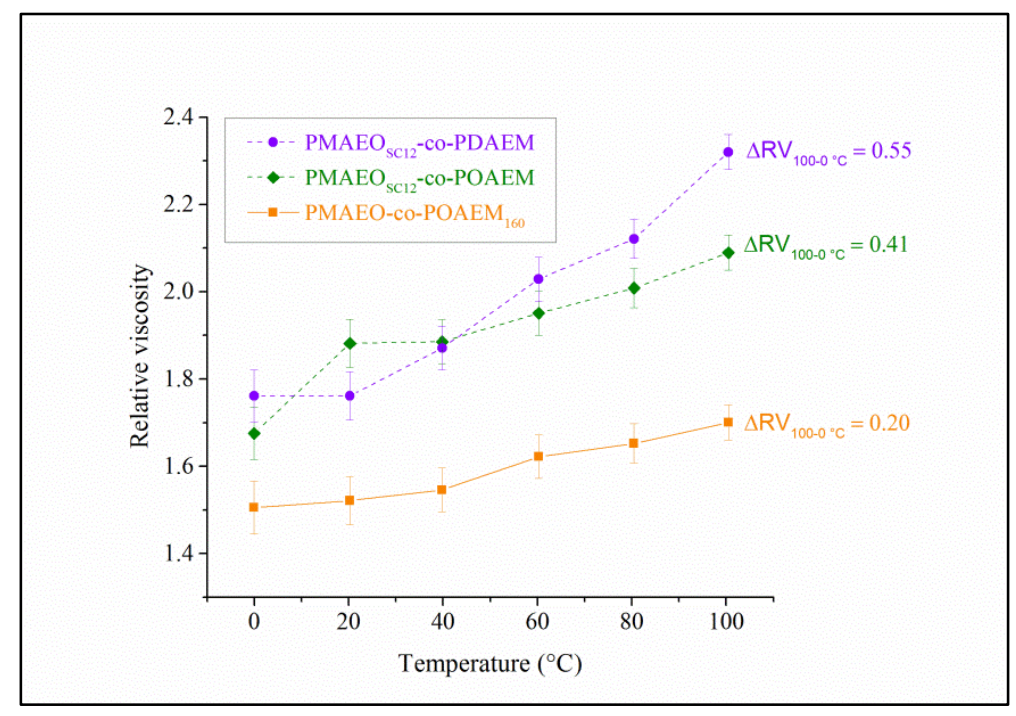

Figure 11: RV versus temperature for copolymers containing a moiety with an additional grafted C-12 chain length (5 wt. \% in OTO)

\subsubsection{Viscosity Index calculations}

Viscosity Index of OTO-copolymer blends were measured according to the ASTM D227010. This value is mainly used by industrial to evaluate the viscosity-temperature dependency of a lubricant. The higher the VI of a lubricant, the lower change in viscosity with temperature.

The results of the VI calculations confirmed the relationship between the coil polymer expansion in oil with temperature and the polymer efficiency as viscosity index improver. As it shown in Table 3, the higher the coil expansion with the temperature, the better the OTO viscosity index improvement. For instance, the addition of PMAEO-co-POAEM 240 in OTO induced the higher increase of relative viscosity with the temperature $\left(\Delta \mathrm{RV}_{100-0}{ }^{\circ} \mathrm{C}=0.55\right)$ and therefore demonstrated the better effect on OTO viscosity index which was increased from 137 to 285 . However, the calculation of viscosity index also revealed the gap of this industrial scale. Despite it was demonstrated that the copolymer PMAEO-co-PMOAEM did not show coil expansion with the temperature, the calculation of the oil-copolymer blend viscosity index revealed that the copolymer was still able to improve OTO's VI from 156 to 200. Thus, the calculation of VI is not enough as argument to confirm the nature of the additive as viscosity index improver since thickeners can also increase the VI. ${ }^{21}$

Table 3: Viscosity index data of OTO blended with 5 wt. \% of copolymers 


\begin{tabular}{|c|c|c|c|c|}
\hline OTO + 5 wt. $\%$ of copolymer & $\begin{array}{l}\mathrm{KV} 40{ }^{\circ} \mathrm{C} \\
\left(\mathrm{mm}^{2} \cdot \mathrm{s}^{-1}\right)\end{array}$ & $\begin{array}{c}\mathrm{KV} 100{ }^{\circ} \mathrm{C} \\
\left(\mathrm{mm}^{2} \cdot \mathrm{s}^{-1}\right)\end{array}$ & Viscosity Index & $\Delta \mathbf{R V}_{100-0}{ }^{\circ} \mathrm{C}$ \\
\hline -- & 26.5 & 5.58 & 156 & - \\
\hline PMAEO-co-PDAEM & 38.40 & 9.50 & 245 & 0.25 \\
\hline PMAEO-co-PMOAEM & 44.47 & 9.29 & 200 & 0 \\
\hline PMAEO $_{\text {SC12} \text {-co-POAEM }}$ & 44.10 & 11.62 & 270 & 0.41 \\
\hline PMAE $_{\text {SC12 }_{2} \text { co-PDAEM }}$ & 46.75 & 12.96 & 285 & 0.55 \\
\hline PMAEO-POAEM $_{110}$ & 38.12 & 8.74 & 220 & 0.13 \\
\hline PMAEO-POAEM $_{160}$ & 39.81 & 9.53 & 235 & 0.20 \\
\hline PMAEO-co-POAEM $_{240}$ & 51.05 & 13.84 & 285 & 0.55 \\
\hline
\end{tabular}

\subsubsection{Rheological behavior of oil-copolymer blends at low temperature}

The triglyceride oil used in this study has the advantage to display a low pour point at -30 ${ }^{\circ} \mathrm{C}$ which means that the lube oil can flow properly until this specific temperature without requiring yield stress. This rheological behavior is characteristic of a Newtonian fluid. In order to confirm that the addition of $5 \mathrm{wt}$ \% FA-based copolymers in OTO did not disturb the oil cold flow properties, the rheological behavior of OTO and oil-copolymer blends which have demonstrated the best VII properties have been studied at $-30{ }^{\circ} \mathrm{C}$. According to Figure 12, the addition of PMAEO-co-POAEM 240 , PMAEO-co-PDAEM and PMAEO SC12-co- $_{2}$ PDAEM induced a non-negligible effect on OTO viscosity at $-30{ }^{\circ} \mathrm{C}$. For instance, at $200 \mathrm{~s}^{-1}$, the dynamic viscosity of oil-PMAEO-co-POAEM 240 blend is $1065 \mathrm{mPa}^{-1} \mathrm{~s}^{-1}$ while $630 \mathrm{mPa}^{-1}$ for pure OTO. This improvement of oil'viscosity may induce some issues on the oil flow at low temperature. 


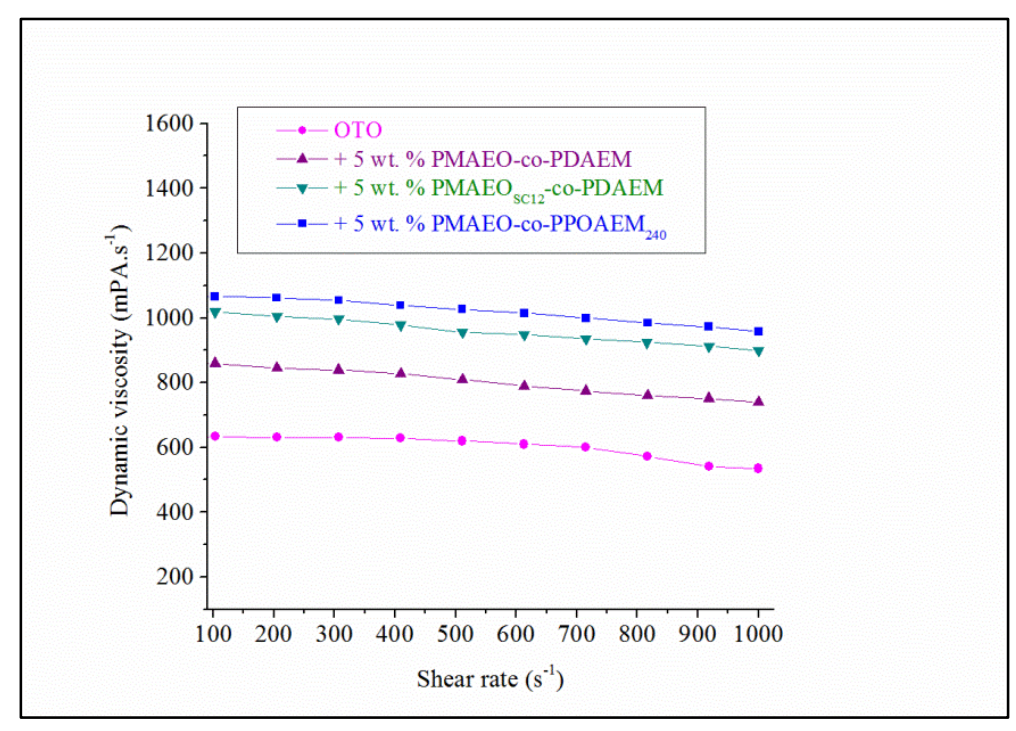

Figure 12: Dynamic viscosity versus shear rate of OTO and OTO-copolymer blends at $-30{ }^{\circ} \mathrm{C}$

However, despite this improvement of oil viscosity with the addition of FA-based copolymers, the Newtonian behavior of OTO was not affected. Indeed, as illustrated in Figure 13, the dynamic viscosity remained approximatively constant with the shear rate for all oilcopolymer blends which is characteristic of Newtonian Fluid. Moreover, the plot of shear stress versus shear rate showed a straight line that passed through the origin with a constant slope for all formulations (Figure 13). This behavior is also characteristic of Newtonian fluids as they do not require any yield stress to flow (intercept of $\mathrm{y}$-axis closed to zero $\mathrm{Pa}$ ). Therefore, it can be deduced that blended OTO with FA-based copolymer additives did not negatively affect the oil flow behavior at $-30{ }^{\circ} \mathrm{C}$.

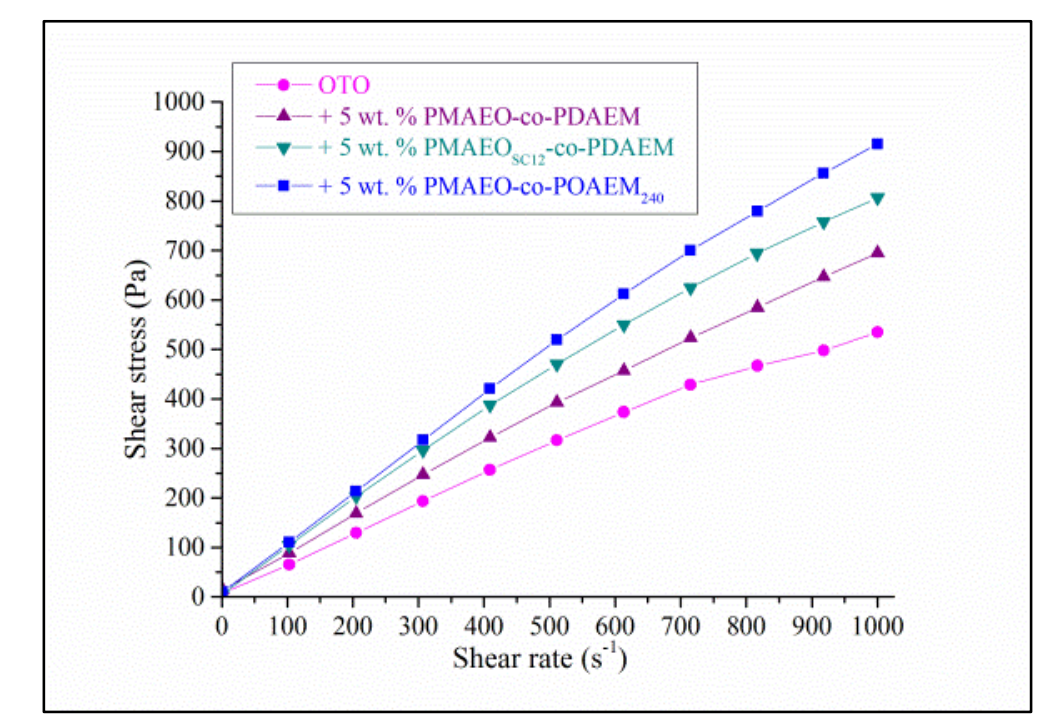

Figure 13: Shear stress vs shear rate of OTO and OTO- copolymer blends at $-30{ }^{\circ} \mathrm{C}$ 


\section{Conclusion}

This work reported the preparation of suitable VII derived from FA for a biolubricant organic triglyceride oil (OTO). Those additives were synthesized by free radical copolymerization of poorly oil-miscible fatty amide methacrylate comonomers and oil-soluble fatty ester methacrylate comonomers (MAEO and $\mathrm{MAEO}_{\mathrm{SC} 12}$ ) with a feed monomers molar ratio of 50:50. The resulting FA-based copolymers were blended at 5 wt. \% in OTO and then evaluated as VII. The results indicated that FA-based the copolymer containing $-\mathrm{NH}_{3}$ have shown a similar thickening power with increasing temperature while those containing $-\mathrm{NH}$ functions were able to have more influence on oil viscosity at high than at low temperatures revealign their high VII properties. Their performance as VII was mostly related to the formation of $\mathrm{H}$-bonding through $-\mathrm{NH}$ functions inducing high polarity at low temperature which decreased the oil copolymer solubility in the hydrophobic oil. In the meantime, increasing temperature have improved the copolymer-OTO interactions through their respective aliphatic chains inducing more effect on oil viscosity at higher temperature. The improvement of the copolymer thickening power in oil with increasing temperature was optimized by increasing the copolymer molecular weight, dispersity, the concentration, the aliphatic pendant chain length of the polymer moieties or by using $\mathrm{MAEO}_{\mathrm{SC} 12}$ instead of MAEO as oil-soluble monomer. Finally, the rheological behavior of OTO with the best VII FA-based copolymers indicated that the additives did not disturb the initial Newtonian behavior of the pure oil at $-30{ }^{\circ} \mathrm{C}$. Hence, the high cold flow properties of formulations coupled with the good thermal stability of FA-based copolymers (up to approximatively 280 ${ }^{\circ} \mathrm{C}$ ) demonstrated that the synthesized FA-based copolymers can be used over a wide range of operating temperatures. Therefore, considering the current environmental context, the use of those novel biobased viscosity index improvers in OTO represent a promising "green" lubricant formulation displaying both excellent cold flow properties and high viscosity index.

\section{Acknowledgment}

This work was performed, in partnership with the SAS PIVERT, within the frame of the French Institute for the Energy Transition (Institut pour la Transition Energétique (ITE) P.I.V.E.R.T. (www.institut-pivert.com) selected as an Investment for the Future ("Investissements d'Avenir"). This work was supported, as part of the Investments for the 
Future, by the French Government under the reference ANR-001-01. The authors have declared no conflict of interest.

\section{References}

(1) Ahmed, M. R.; Mohammed, A. H. A.-K.; A.hamad, M. Synthesis, Characterization and Performance Evaluation of Poly Octadecyl Methacrylate and Poly Octadecyl Methacrylate-CoMethylmethacrylate as an Additive for Lubricating Oil. IOSR Journal of Applied Chemistry 2017, 10 (04), 50-58.

(2) Akhmedov, A. I.; Buniyat-Zade, I. A. Principles of Synthesis of Viscosity Index Improvers of the Poly(Alkyl Methacrylate) Type. Chem Technol Fuels Oils 1993, 29 (4), 207-212.

(3) Almeida, A. P. P.; Oliveira, A. P. L. R. de; Erbetta, C. D. C.; Sousa, R. G. de; Freitas, R. F. de S.; Silva, M. E. S. R. e. Rheological Study of Polymers Used as Viscosity Index Improvers for Automotive Lubricant Oils. Journal of Modern Physics 2014, 05 (12), 1085-1093.

(4) Neveu, C. D.; Sondjaja, R.; Stöhr, T.; Iroff, N. J. Lubricant and Fuel Additives Based on Polyalkylmethacrylates. In Polymer Science: A Comprehensive Reference; Elsevier, 2012; pp 453-478.

(5) Selby, T. W. The Non-Newtonian Characteristics of Lubricating Oils. A $S L E$ Transactions 1958, 1 (1), 68-81.

(6) Sonntag, N. O. V. Straight-Chain Fatty Acids from Alcohols, Olefins, and Ziegler Intermediates. J Am Oil Chem Soc 1968, 45 (1), 14-16.

(7) Tomašek, L.; Jukić, A.; Janović, Z. Copolymerization of Methyl Methacrylate and Dodecyl Methacrylate Initiated by Bifunctional Peroxide. Acta chimica Slovenica 2005, 52 (3), 224-229.

(8) Characterization of Fatty Acids Composition in Vegetable Oils by Gas Chromatography and Chemometrics. Analytica Chimica Acta 1998, 358 (2), 163-175.

(9) McNutt, J.; He, Q. (Sophia). Development of Biolubricants from Vegetable Oils via Chemical Modification. Journal of Industrial and Engineering Chemistry 2016, 36, 112.

(10) Ghosh, P.; Karmakar, G. Evaluation of Sunflower Oil as a Multifunctional Lubricating Oil Additive. International Journal of Industrial Chemistry 2014, 5 (1), 7-17.

(11) Ghosh, P.; Hoque, M.; Karmakar, G. Castor Oil as Potential Multifunctional Additive in the Formulation of Eco-Friendly Lubricant. Polym. Bull. 2018, 75 (2), 501-514.

(12) Ghosh, P.; Upadhyay, M. Isodecyl Acrylate - Olive Oil Copolymers as Potential Biodegradable Additive for Lubricating Oil. J Polym Res 2016, 23 (5), 100-120.

(13) Montero de Espinosa, L.; Meier, M. A. R. Plant Oils: The Perfect Renewable Resource for Polymer Science?! European Polymer Journal 2011, 47 (5), 837-852.

(14) Ghosh, P.; Das, T.; Karmakar, G.; Das, M. Evaluation of Acrylate-Sunflower Oil Copolymer as Viscosity Index Improvers for Lube Oils. International Journal of Industrial Chemistry 2011, 3 (3), 547-556.

(15) Ghosh, P. Soybean Oil as a Biocompatible Multifunctional Additive for Lubricating Oil. American Chemical Society 2014, 19-25.

(16) Nasser, R. Jojoba Polymers As Lubricating Oil Additives. Petroleum and Coal 2015, $2(57), 120-129$. 
(17) Lomège, J.; Negrell, C.; Robin, J.-J.; Lapinte, V.; Caillol, S. Fatty Acid-Based Methacrylate Polymers as Viscosity Modifiers for Mineral Oils. Green Materials 2018, 6 (3), 97-107.

(18) Lomège, J.; Negrell, C.; Robin, J.-J.; Lapinte, V.; Caillol, S. Oleic Acid-Based Poly(Alkyl Methacrylate) as Bio-Based Viscosity Control Additive for Mineral and Vegetable Oils. Polymer Engineering \& Science 2018, No. 0.

(19) Bennekom, A. C. M. van; Bennekom, A. C. M. van; Willemsen, P. a. a. T.; Willemsen, P. a. a. T.; Gaymans, R. J. Amide-modified poly(butylene terepthalate): thermal stability. Polymer 1996, 37 (24), 5447-5459.

(20) Covitch, M. J.; Trickett, K. J. How Polymers Behave as Viscosity Index Improvers in Lubricating Oils. Advances in Chemical Engineering and Science 2015, 05 (02), 134151.

(21) Martini, A.; Ramasamy, U. S.; Len, M. Review of Viscosity Modifier Lubricant Additives. Tribol Lett 2018, 66 (2), 58-72. 